\title{
ON ASSOCIATIVE PRODUCTS OF GROUPS $\left({ }^{1}\right)$
}

\author{
BY \\ RUTH REBEKKA STRUIK
}

1. Introduction( $\left.{ }^{2}\right)$. A. G. Kurosh [1, p. 323] has formulated the following problem: The free and the direct product of a set of groups are algebraic operations on these groups which have the following properties:

(a) the operations are commutative;

(b) the operations are associative;

(c) the product contains subgroups which generate the product;

(d) these subgroups are isomorphic to the original groups;

(e) the intersection of a given one of these subgroups with the normal subgroups generated by the rest of these subgroups is the identity. The question arises, are there any other products, other than the free and the direct product, which also have these properties?

The answer to this question is yes. Golovin (see [3]) discovered a denumerable number of such products which he called nilpotent products. We give in this paper another set of such products which include nilpotent products as a special case. S. Moran [5] has also produced another set of products which he called verbal products.

To the properties (a) through (e) above, S. MacLane has added

(f) (MacLane's postulate): if each of these subgroups (in the product) is replaced by a factor group of the same subgroup, then the resulting group is the same as the product of the factor groups.

In his paper, Golovin introduced what he called regular products and fully regular products. Regular products are those products which satisfy conditions (c) and (e); Golovin showed that they necessarily satisfy (d). Fully regular products satisfy conditions (a), (b), (c), (d), and (e). Golovin asks, but does not answer, the question as to whether all regular products are associative. The answer will be given here in the negative. Verbal products (which include nilpotent products) satisfy MacLane's postulate. A fully regular product which does not satisfy MacLane's postulate will be given here; hence MacLane's postulate is independent of (a) through (e).

Presented to the Society, February 26, 1955; received by the editors March 21, 1955 and, in revised form, June 7, 1955.

(1) Part of the present paper is contained in the author's doctoral dissertation in the department of mathematics at New York University. Professor Wilhelm Magnus suggested the problem and guided the work to completion; his aid is gratefully acknowledged.

(2) The formulation and significance of the problem given in this introduction are taken from Golovin's paper [3]. 
In this paper regular products are investigated further, and several nonassociative, commutative products are produced.

The significance of this problem is that its solution may lead to a more detailed classification of groups; for example, by means of direct products, all Abelian groups with a finite number of generators can be classified. It would also be a tool for the creation of new groups or new classes of groups.

The following questions are still open:

(i) Are there any more fully regular products satisfying MacLane's postulate other than those already mentioned here? If so, how can they be classified?

(ii) How can all regular products be classified? Given a regular product, how can one decide if it is fully regular?

(iii) Given a group, defined by means of a set of generators and some defining relations, how can one tell whether or not it is a fully regular product of its subgroups?

In the second section of this paper, definitions and notation are given, and theorems proved in Golovin's paper are stated. Thus it should be possible to follow this paper without previous study of Golovin's work, but obviously such study would be helpful.

In the third section, theorems needed in the fourth and fifth sections are proved, and an important theorem due to $\mathrm{W}$. Magnus is stated. Theorem 3.7 is of particular importance, since it is the key identity used to prove that Golovin's nilpotent products are a special case of the associative products given in $\$ 5$. It is also of intrinsic interest, since it gives a relation between members of several lower central series of a free product.

In the fourth, fifth and sixth section, the final results are stated and proved; in the fourth section, the results on nonassociative products; in the fifth section, the results on associative products. A general method for proving a large number of regular products nonassociative is given in the second proof of Theorem 4.8. Notations and conventions are introduced there which are necessary in understanding the proofs of Theorems 4.9 through 4.11.

In the sixth section MacLane's postulate is discussed, and a fully regular product is given which does not satisfy this postulate. The relation between the work of S. Moran and the results of this paper is summarized.

2. Notations, definitions, and theorems proved elsewhere. The following notations and definitions will be used throughout:

$G=\cap_{i} A_{i}$ means that $G$ is the intersection of the sets (groups) $A_{i}$.

$G \cong H$ means the group $G$ is isomorphic to the group $H$.

$\mathfrak{N}^{\sigma}[A]$ denotes the normal subgroup of $G$ generated by the set (group) $A$. If there is no confusion, this will be denoted by $\mathfrak{N}[A]$ alone. If $H$ is a subgroup of $G$, and both $\mathfrak{N}^{B}[A]$ and $\mathfrak{N}^{G}[A]$ are under discussion, then $\mathfrak{N}[A]$ will refer to $\mathfrak{N}^{G}[A]$.

$C=A / B$ means that the group $C$ is the factor group of $A$ modulo $B$. 
$G=\left\{A_{\alpha}, \alpha \in M\right\}$ means that $G$ is generated by the subsets (subgroups) $A_{\alpha}$ where the index $\alpha$ runs over the set $M$. Similarly, $G=\{A, B, \cdots\}$ means that $G$ is generated by $A, B, \cdots$.

$G=\prod_{\alpha \in M} A_{\alpha}$ means that $G$ is the product of its normal divisors $A_{\alpha}$; however, one of the $A_{\alpha}$ may not be normal. Similarly $G=A \cdot B \cdot C$ means that $G$ is the product of its subgroups $A, B, C$, two of which are normal.

$G=\prod_{\alpha \in M}{ }^{*} A_{\alpha}$ means that $G$ is the free product of the $A_{\alpha}$. Similarly $G=A * B * C$ means $G$ is the free product of $A, B$, and $C$.

$G=\prod_{\alpha \in M} \times A_{\alpha}$ means that $G$ is the direct product of the groups $A_{\alpha}$. Similarly $G=A \times B \times C$ means that $G$ is the direct product of the groups $A, B$, and $C$.

$(x, y)=x^{-1} y^{-1} x y$ denotes the commutator of the group elements, $x, y$.

$(A, B)$ denotes the group generated by $(a, b)$ for all $a \in A$ and $b \in B$.

${ }^{\circ} A=A,{ }^{k} A=\left({ }^{k-1} A, A\right)$, where $k$ is a non-negative integer.

${ }_{0} A_{G}=\mathfrak{R}^{G}[A],{ }_{k} A_{G}=\left({ }_{k-1} A_{G}, G\right)$. This is known as the lower central series determined by $A$. If there is no danger of confusion, ${ }_{k} A$ will be used. If $H$ is a subgroup of $G$ and both ${ }_{k} A_{H}$ and ${ }_{k} A_{G}$ are under discussion, then ${ }_{k} A$ will refer to ${ }_{k} A_{G}$.

$G=\prod_{\alpha \in M} \circ A_{\alpha}$ is a regular product of the groups $A_{\alpha}$ if

(a) $G=\left\{A_{\alpha}, \alpha \in M\right\}$;

(b) $A_{\alpha} \cap \mathfrak{N}\left[B_{\alpha}\right]=1$, where $B_{\alpha}=\left\{A_{\beta}, \beta \neq \alpha, \beta \in M\right\}$ and 1 is the identity element of $G$.

Fully regular products are regular products which are associative and commutative. This means that if $A \circ B$ is a fully regular product of $A$ and $B$, then $A \circ B \cong B \circ A$ under the obvious mapping. If any three groups $A, B$, and $C$ are given, then $(A \circ B) \circ C \cong A \circ(B \circ C)$ under the obvious mapping (of $A \rightarrow A, B \rightarrow B, C \rightarrow C$ ). $A(k) B$ is the kth nilpotent product of $A$ and $B$ if

$$
A(k) B=(A * B) / k(A, B)_{A} *_{B} .
$$

A free associative ring, $R$, with generators $x_{1}, \cdots, x_{r}$ over the integers is a ring generated by the $x_{i}$ and the integers with the following properties:

(i) all the usual ring operations hold except that multiplication is noncommutative;

(ii) the integers commute with every element;

(iii) no other relations hold between elements of the ring other than (i) and (ii);

(iv) a typical element of $R$ is a finite or infinite sum of the form

$$
n+\sum n\left(\alpha_{1}, \alpha_{2}, \cdots, \alpha_{m} ; a_{1}, a_{2}, \cdots, a_{m}\right) x_{\alpha_{1}}^{a_{1}} x_{\alpha_{2}} a_{2} \ldots x_{\alpha_{m}}^{a_{m}}
$$

where $n, \alpha_{i}, a_{i}$ and $n\left(\alpha_{1}, \alpha_{2}, \cdots, \alpha_{m} ; a_{1}, a_{2}, \cdots, a_{m}\right)$ are integers $\left(1 \leqq \alpha_{i} \leqq r\right)$, and the summation extends over all possible combinations. Questions of convergence do not enter here, since the summation is purely formal.

In a free associative ring $R,[x, y]=x y-y x$. 
$\mathrm{G} n ; m$ will refer to a theorem or identity proved by Golovin (see [3]) in chapter $n$, section $m$ of his paper. For example, G I; 3.1 will refer to Chapter I, §3.1. $n . m$ will refer to the $m$ th theorem proved in $\S n$ of this paper.

M1 will refer to a theorem proved by Magnus.

The following theorems and identities are stated by Golovin in his paper. Not all of them are original with Golovin. Many are proved in his paper.

$\mathrm{G} \mathrm{I} ; 2.1 .1(x, y)=(y, x)^{-1}$.

G I ; 2.1.2 $(x y, z)=y^{-1}(x, z) y(y, z)=(x, z)((x, z), y)(y, z)$.

G I ; 2.1.3 $(x, y z)=(x, z) z^{-1}(x, y) z=(x, z)(z,(y, x))(x, y)$.

$\mathrm{G} \mathrm{I} ; 2.3(A, B)=(B, A)$.

G I; 2.4.1 If $A$ is a subgroup of $G$, then $(G, A) \leqq A$ if and only if $A$ is normal.

G I 2.4 .2 If $A \leqq B$ and $C \leqq D$, then $(A, C) \leqq(B, D)$.

$\mathrm{G} \mathrm{I} ; 2.4 .3(A, B) \leqq \mathfrak{N}[A] \cap \mathfrak{N}[B]$.

$\mathrm{G}$ I; 2.4.5 $\mathfrak{N}[(A, B)] \leqq(\mathfrak{R}[A], \mathfrak{R}[B])$, and the inequality may hold.

$\mathrm{G} I ; 3.1$ If $G=\{A, B\}$, then $(A, B)=\mathfrak{R}[(A, B)]=(\mathfrak{R}[A], \mathfrak{R}[B])$.

$\mathrm{G} \mathrm{I} ; 3.5$ If $G=\{A, B\}$, then $(G, A)=(A, A)(A, B)$.

G I; 3.6 If $N$ is normal in $G$, and $A$ is an arbitrary subgroup of $G$, and $S_{A}$ is a system of generators of $A$, then $(N, A)=\left(N, S_{A}\right)$.

G I ; 4.3 If $N$ and $M_{\alpha}$ are normal subgroups of a group $G$, and $A$ is an arbitrary subgroup, then

$$
\left(A \cdot \prod_{\alpha} M_{\alpha}, N\right)=(A, N) \prod_{\alpha}\left(M_{\alpha}, N\right) .
$$

G I; 4.8 If $A, B$, and $C$ are normal subgroups of $G$, then

$$
((A, B), C) \leqq((B, C), A)((C, A), B) .
$$

G I; 5.3 If $A_{\alpha}$ are arbitrary subgroups of $G$, then

$$
{ }_{m}\left\{A_{\alpha}\right\}=\prod_{\alpha} A_{\alpha}, \quad m=0,1,2, \cdots
$$

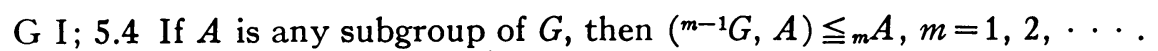

G II; 1.2 Let $G=\left\{A_{\alpha}, \alpha \in M\right\}$, where $M$ is an ordered set. Then $G$ is a regular product of the $A_{\alpha}$ if and only if every element $g$ of $G$ can be written in the form

$$
g=a_{\alpha_{1}} a_{\alpha_{2}} \cdots a_{\alpha_{n}} u
$$

where $a_{\alpha_{i}} \in A_{\alpha_{i}}, u \in\left(\mathfrak{R}\left[A_{\alpha}\right]\right)=\left\{\left(\mathfrak{N}\left[A_{\alpha}\right], \mathfrak{N}\left[A_{\beta}\right]\right), \alpha \neq \beta\right\}$, and $\alpha_{1}<\alpha_{2}<\cdots$ $<\alpha_{n}$. The $a_{\alpha_{i}}$ are unique up to factors equal to 1 (the identity of the group(s)).

$\mathrm{G}$ II $; 1.14$ If $G=A * B$, then $(A, B)$ is a free group generated by $(a, b)$, $a \in A, b \in B$.

$\mathrm{G}$ II; 5.7 All nilpotent products of groups are fully regular products.

G II; 6.3 If $G=A(k) B$, and $A$ and $B$ are finite, then $G$ is finite. 
3. Preliminary theorems. In this section, we prove theorems needed in $\S \S 4$ and 5. Theorem 3.3 (for which Theorems 3.1 and 3.2 are needed) is a key theorem in proving the associativity of the products introduced in $\$ 5$. Theorems 3.4, 3.5, and 3.6 are needed to prove Theorem 3.7 whose significance is mentioned in $\$ 1$. Theorem M1, proved by W. Magnus, and Theorem 3.8 are the heart of a general method used in $\$ 4$ to prove that a large number of products are not associative.

TheOREM 3.1. Let $P$ and $Q$ be subgroups of the group $G$. Then $\left({ }_{i} P,{ }_{i} Q\right)$ $\leqq_{i+j+1} P \bigcap_{i+j+1} Q$, and in particular $\left({ }_{i} P,{ }_{j} G\right) \leqq_{i+j+1} P$.

(Comment. This is a generalization of G I; 5.4.)

Proof. This follows almost immediately from G I; 5.4, for since ${ }_{j} G={ }^{i} G$,

$$
\begin{aligned}
& \left({ }_{i} P,{ }_{j} G\right) \leqq{ }_{j+1}\left({ }_{i} P\right)={ }_{i+i+1} P ; \\
& \left({ }_{i} P,{ }_{i} Q\right) \leqq\left({ }_{i} P,{ }_{i} G\right) \leqq{ }_{i+j+1} P .
\end{aligned}
$$

(Use is made of $\mathrm{G} \mathrm{I}$; 2.4.2.)

Similarly, $\left({ }_{i} P,{ }_{j} Q\right) \leqq_{i+j+1} Q$. q.e.d.

TheOREM 3.2. Let $P$ and $Q$ be arbitrary subgroups of a group $G$, and $M$ and $N$ normal subgroups of $G$. Then

$$
(P N, Q M) \leqq \mathfrak{R}[(P, Q)(P, M)(Q, N)](N, M) .
$$

Comment. Note that $(N, M)$ is normal since $N$ and $M$ are.

Proof. $(P N, Q M)$ is generated by $(p n, q m)$ where $p \in P, q \in Q, n \in N$, $m \in M$. This is where normality of $N$ and $M$ are used. Using G I; 2.1.3 and G I; 2.1.2, we have

$(p n, q m)=(p n, m) m^{-1}(p n, q) m=\left[n^{-1}(p, m) n\right](n, m)\left[m^{-1}\left[n^{-1}(p, q) n\right](n, q) m\right]$.

Since $\mathfrak{R}[(P, Q)(P, M)(Q, N)](N, M)$ is normal, the proof is finished.

Theorem 3.3. Let $F=G * H$, and let $P$ be a subgroup of $G$; then

$$
{ }_{k} P_{F} \leqq{ }_{k} P_{G}\left({ }_{k} G \cap{ }_{k} H\right), \quad k=0,1, \cdots .
$$

Proof. The proof is by induction on $k$.

$k=0$ : We have to show that $\mathfrak{N}[D] \leqq \mathfrak{N}^{\sigma}[P](\mathfrak{R}[H] \cap \mathfrak{N}[G]) . \mathfrak{N}[P]$ is generated by elements of the form $f^{-1} p f$, where $f \in F, p \in P$. Since free products are regular, by G II; 1.2 , every element of $F$ can be written in the form $f=g h u$, where $g \in G, h \in H$ and $u \in(G, H) . f^{-1} p f=p(p, f) \in \mathfrak{N}^{G}[P](P, F)$. Therefore, we want to show that $(p, g h u) \in \mathfrak{N}^{G}[P](\mathfrak{N}[H] \cap \mathfrak{N}[G])$ for all $g \in G, h \in H$, $u \in(G, H) .(p, g) \in \mathfrak{N}^{\sigma}[P]$ by $\mathrm{G} I ; 2.4 .3 .(p, h) \in(G, H) \leqq \mathfrak{R}[G] \cap \mathfrak{R}[H]$ by $\mathrm{G} I$; 2.4.3. Similarly by also using $\mathrm{G} I ; 3.1,(p, u) \in \Re[G] \cap \Re[H]$. Using $\mathrm{G} \mathrm{I}$; 2.1.3,

$$
\begin{aligned}
(p, g h) & =(p, h)(h,(g, p))(p, g), \\
(p, g h u) & =(p, u)(u,(g h, p))(p, g h) .
\end{aligned}
$$


Since $(h,(g, p)) \in(H, G)$ and $(u,(g h, p)) \in_{1}(G, H) \leqq(G, H) \leqq \mathfrak{N}[G] \cap \mathfrak{N}[H]$, we have proved the theorem for $k=0$.

Suppose true for $k-1 .{ }_{k} P=\left({ }_{k-1} P, F\right)$. By G II ; 1.2 , we have to show that $(r, g h u) \in_{k} P_{G}\left({ }_{k} H \cap_{k} G\right)$ for all $r \in_{k-1} P \leqq_{k-1} G, g, h, u$ as above. By induction, $r={ }_{k-1} p n$, where ${ }_{k-1} p \in \in_{k-1} P_{G}, n \in \in_{k-1} G \cap_{k-1} H$. Using G I; 2.1.2, and Theorem $3.1,(r, g)=\left({ }_{k-1} p n, g\right)=\left({ }_{k-1} p, g\right)\left(\left({ }_{k-1} p, g\right), n\right)(n, g) \in_{k} P_{G} \cdot\left({ }_{k} G,{ }_{k-1} H\right) \cdot\left({ }_{k-1} H, G\right)$ $\leqq_{k} P_{G}\left({ }_{k} H \cap{ }_{k} G\right)$, where use has also been made of the fact that ${ }_{k-1} U \geqq_{k} U$ for any subgroup $U$. Similarly, $(r, h) \in\left({ }_{k-1} G, H\right) \leqq_{k} G \cap_{k} H ;(r, u) \in\left({ }_{k-1} G,{ }_{o} H\right)$ $\leqq_{k} \mathfrak{R}[G] \cap_{k} \mathfrak{N}[H]$. Using G I; 2.1.3, we obtain

$$
(r, g h)=(r, h)(h,(g, r))(r, g)
$$

and

$$
(r, g h u)=(r, u)(u,(g h, r))(r, g h) .
$$

But $(h,(g, r)) \in\left(H,{ }_{k} G\right) \leqq{ }_{k} G \cap{ }_{k} H$ and $(u,(g h, r)) \in\left({ }_{o} H,{ }_{k} G\right) \leqq{ }_{k} G \cap{ }_{k} H$. q.e.d.

Theorem 3.4. If $G=\{A, B\}$, then $(k \mathfrak{N}[A], \mathfrak{N}[B]) \leqq{ }_{k}(A, B)$.

Comment. This is similar to G II; 4.5 .

Proof. The proof is by induction on $k$. For $k=0$, use G I; $3.1:\left(^{\circ} \mathfrak{R}[A]\right.$, $\mathfrak{R}[B])=(\mathfrak{R}[A], \mathfrak{R}[B])={ }_{\mathrm{o}}(A, B)$. Suppose true for $k-1$; then using $\mathrm{G} \mathrm{I} ; 4.8$, the induction hypothesis, G I; 3.1, and Theorem 3.1, and various definitions,

$$
\begin{aligned}
\left({ }^{k} \mathfrak{N}[A], \mathfrak{N}[B]\right) & =\left(\left({ }^{k-1} \mathfrak{N}[A], \mathfrak{N}[A]\right), \mathfrak{N}[B]\right) \\
& \leqq\left(\left({ }^{k-1} \mathfrak{N}[A], \mathfrak{N}[B]\right), \mathfrak{N}[A]\right)\left((\mathfrak{N}[A], \mathfrak{N}[B]),{ }^{k-1} \mathfrak{N}[A]\right) \\
& \leqq\left({ }_{k-1}(A, B), G\right)\left((A, B),{ }^{k-1} G\right) \leqq{ }_{k}(A, B) .
\end{aligned}
$$

Theorem 3.5. If $R, S, T$, and $S * T$ are subgroups of $G$, then

$$
\mathfrak{N}[(R, S * T)]=\mathfrak{N}[(R, S)(R, T)] \leqq(\mathfrak{R}[R], \mathfrak{N}[S])(\mathfrak{N}[R], \mathfrak{N}[T])
$$

Proof. The inequality follows from G I ; 2.4.5. $\mathfrak{R}[(R, S * T)] \geqq \mathfrak{R}[(R, S)$ $\cdot(R, T)]=V$ is trivial. The opposite inequality is proved by induction on the length of an element in $S * T .(r, s),(r, t) \in V$ for all $r \in R, s \in S, t \in T$, obviously. Suppose $u$ is an element of $S * T$ of length $n$. Then by G I; 2.1.3, $(r, u s)=(r, s) s^{-1}(r, u) s$ which $\in V$ by induction and the normality of $V$. Similarly $(r, u t) \in V$. q.e.d.

TheOREM 3.6. Let $G=A * B$, then ${ }_{k} A \leqq{ }^{k} \mathfrak{R}[A]_{k-1}(A, B), k=0,1, \cdots$, where ${ }_{-1}(A, B)=1$.

Proof. The proof is by induction. For $k=0, \propto A=\mathfrak{N}[A]={ }^{\circ} \mathfrak{N}[A]$. For $k=1,{ }_{1} A=(A, G)=(A, A)(A, B) \leqq 1 \mathfrak{R}[A]_{\circ}(A, B)$, where $\mathrm{G} \mathrm{I} ; 3.5$ and some definitions have been used. Suppose true for $k$; then using the induction hypothesis, G I ; 4.3, Theorem 3.5, Theorem 3.4 , and various definitions, we have 


$$
\begin{aligned}
{ }_{k+1} A & =\left({ }_{k} A, G\right) \leqq\left({ }^{\mathfrak{N}}[A]_{k-1}(A, B), G\right)=\left({ }^{k} \mathfrak{N}[A], A * B\right)\left({ }_{k-1}(A, B), G\right) \\
& \leqq\left({ }^{k} \mathfrak{N}[A], \mathfrak{N}[A]\right)\left({ }^{k} \mathbb{N}[A], \mathfrak{N}[B]\right)_{k}(A, B) \leqq{ }^{k+1} \mathfrak{N}[A]{ }_{k}(A, B) .
\end{aligned}
$$

TheOREM 3.7. Let $G=A * B$, then ${ }_{k}(A, B)=\prod_{m+n=k}\left({ }_{m} A,{ }_{n} B\right)=\left({ }_{0} A,{ }_{k} B\right)$ $\left({ }_{0} B,{ }_{k} A\right)$.

Proof. To prove the first equality, we show (i) ${ }_{k}(A, B) \leqq \prod_{m+n=k}\left({ }_{m} A,{ }_{n} B\right)$ and then (ii) ${ }_{k}(A, B) \geqq\left({ }_{m} A,{ }_{n} B\right)$ for $m+n=k$.

(i) The proof is by.induction on $k$. For $k=0, \mathrm{G} \mathrm{I} ; 3.1$ suffices. Suppose true for $k$. Then using the induction hypothesis, G I ; 4.3, G I ; 4.8, and various definitions, we have

$$
{ }_{k+1}(A, B)=\left({ }_{k}(A, B), G\right) \leqq\left(\prod_{m+n=k}\left({ }_{m} A,{ }_{n} B\right), G\right)=\prod_{m+n=k}\left(\left({ }_{m} A,{ }_{n} B\right), G\right) .
$$

But

$$
\left(\left({ }_{m} A,{ }_{n} B\right), G\right) \leqq\left(\left({ }_{m} A, G\right),{ }_{n} B\right)\left(\left({ }_{n} B, G\right),{ }_{m} A\right)=\left({ }_{m+1} A,{ }_{n} B\right)\left({ }_{n+1} B,{ }_{m} A\right) .
$$

This proves (i). To prove (ii), use induction on $k$. For $k=0, \mathrm{G}$ I; 3.1 suffices. Suppose true for $k$, and let $m+n=k+1$. Then by using $\mathrm{G} \mathrm{I} ; 4.8$, the induction hypothesis and various definitions, we have

$$
\begin{aligned}
\left({ }_{m} A,{ }_{n} B\right) & =\left(\left({ }_{m-1} A, G\right),{ }_{n} B\right) \leqq\left({ }_{m-1} A,\left(G,{ }_{n} B\right)\right)\left(\left({ }_{m-1} A,{ }_{n} B\right), G\right) \\
& \leqq\left({ }_{m-1} A,{ }_{n+1} B\right)\left(_{m+n-1}(A, B), G\right)=\left({ }_{m-1} A,{ }_{n+1} B\right)_{k+1}(A, B) .
\end{aligned}
$$

By repeating this as many times as is necessary, we obtain

$$
\left({ }_{m} A,{ }_{n} B\right) \leqq\left({ }_{0} A,{ }_{k+1} B\right) \cdot{ }_{k+1}(A, B),
$$

and similarly,

$$
\left({ }_{m} A,{ }_{n} B\right) \leqq\left({ }_{k+1} A,{ }_{\circ} B\right)_{k+1}(A, B) .
$$

Now, using Theorem 3.6, G I; 4.3, and Theorem 3.4, we obtain

$$
\begin{aligned}
\left({ }_{k+1} A,{ }_{\circ} B\right) & \leqq\left({ }^{k+1} \mathfrak{N}[A] \cdot{ }_{k}(A, B), \mathfrak{R}[B]\right) \\
& =\left({ }^{k+1} \mathfrak{N}[A], \mathfrak{N}[B]\right)\left({ }_{k}(A, B), \mathfrak{N}[B]\right) \leqq{ }_{k+1}(A, B) .
\end{aligned}
$$

This proves the first equality. To prove the second equality, it is sufficient to show that for $k \geqq 1,{ }_{k}(A, B) \leqq\left({ }_{\circ} A,{ }_{k} B\right)\left({ }_{k} A,{ }_{0} B\right)$ in view of the inequalities proved in the first part of the proof. But ${ }_{k} A=(\cdots(A, G), \cdots, G)$ $\geqq(\cdots((A, B), G), \cdots, G)={ }_{k-1}(A, B)$. Therefore, since all groups considered are normal, with the use of Theorem 3.5,

$$
\begin{aligned}
\left({ }_{k} A,{ }_{\circ} B\right)\left({ }_{\circ} A,{ }_{k} B\right) & \geqq\left({ }_{k-1}(A, B), \mathfrak{N}[B]\right)\left(\mathfrak{N}[A],{ }_{k-1}(A, B)\right) \\
& \geqq\left({ }_{k-1}(A, B), A * B\right)={ }_{k}(A, B) .
\end{aligned}
$$

The following theorem was proved by Magnus (see [4]). 
MI. Let $F$ be a free group with generators $a_{1}, a_{2}, \cdots, a_{n}$; let $R$ be a free associative ring over the integers with generators $x_{1}, x_{2}, \cdots, x_{n}$. Let $a_{i}$ correspond to $1+x_{i}$ and $a_{i}^{-1}$ correspond to $\sum_{j=0}^{\infty}(-1)^{i} x_{i}^{j}$. Then to each $b \in F$ corresponds a unique series of the form

$$
1+\sum_{j=1}^{\infty} P_{j}\left(x_{i}\right)
$$

where $P_{j}\left(x_{i}\right)$ is a polynomial of degree $j$ in the $x_{i}$. (Here $x_{i} x_{k}$ is considered a polynomial of degree two.) Then the image of $F$ in $R$ is a faithful representation of $F$. Let $Z_{1}=F, Z_{k}=\left(Z_{k-1}, F\right)$. Let $D_{k}$ be the set of all $b \in F$ such that $b$ corresponds to a polynomial of the form $1+\sum_{j=k}^{\infty} P_{j}\left(x_{i}\right)$, i.e., $P_{j}\left(x_{i}\right)=0$ for all $j<k$. Then $Z_{k}=D_{k}$. For convenience, we shall write (in the future) $b=1+\sum P_{j}\left(x_{i}\right)$.

THEOREM 3.8. The following identities given here can be proved by direct computation. Let $p, q$ be elements of a free group, and $u_{i}, v_{i}, w_{i}, t_{i}$ elements of degree $i$ of a free associative ring over the integers. Then using the correspondence set up in Theorem MI, let

$$
\begin{aligned}
p & =1+u_{1}+u_{2}+u_{3}+\cdots, \\
q & =1+v_{1}+v_{2}+v_{3}+\cdots, \\
p^{-1} & =1+w_{1}+w_{2}+w_{3}+\cdots, \\
q^{-1} & =1+t_{1}+t_{2}+t_{3}+\cdots ;
\end{aligned}
$$

then

$$
\begin{array}{lc}
u_{1}+w_{1}=0, & v_{1}+t_{1}=0, \\
u_{2}+w_{2}-u_{1}^{2}=0, & v_{2}+t_{2}-v_{1}^{2}=0, \\
u_{3}+w_{3}+u_{1} w_{2}-u_{2} u_{1}=u_{3}+w_{3}-u_{1} u_{2}+w_{2} u_{1}=0, \\
v_{3}+t_{3}+v_{1} t_{2}-v_{2} v_{1}=v_{3}+t_{3}-v_{1} v_{2}+t_{2} v_{1}=0 ;
\end{array}
$$

and

(a) $(p, q)=1+\left[u_{1}, v_{1}\right]+\left(\left[u_{1}, v_{2}\right]+\left[u_{2}, v_{1}\right]+\left(u_{1}+v_{1}\right)\left[v_{1}, u_{1}\right]\right)+\cdots$.

If $p=1+u_{k}+u_{k+1}+\cdots, q=1+v_{1}+v_{2}+\cdots$ where $k \geqq 2$, then

$$
(p, q)=1+\left[u_{k}, v_{1}\right]+\cdots
$$

and

$$
q^{-1} p q=p(p, q)=1+u_{k}+\left(u_{k+1}+\left[u_{k}, v_{1}\right]\right)+\cdots .
$$

$$
\text { If } p=1+u_{2}+u_{3}+\cdots, q=1+v_{2}+v_{3}+\cdots \text {, then }
$$

$$
(p, q)=1+\left[u_{2}, v_{2}\right]+\left(\left[u_{2}, v_{3}\right]+\left[u_{3}, v_{2}\right]\right)+\cdots .
$$


4. Nonassociative products. In this section, several regular products will be defined and proved to be nonassociative.

In his paper Golovin asked, but did not answer, the following question: "Are all regular products associative?" An almost trivial example given in Theorem 4.1 shows that they are not. However, this product is not commutative, and the question arises, are all commutative regular products associative? Theorem 4.2 gives the answer in the negative.

Theorems 4.3 through 4.8 give proofs of the nonassociativity of some commutative regular products. Each of these proofs is a special one, using a method probably not applicable to other nonassociative products. Starting with Theorem 4.8, a method is used which is due to W. Magnus, using Theorem $\mathrm{M} 1$ on the relations between commutator subgroups of a free group and elements of a free associative ring. This method is used to prove the nonassociativity of a large number of commutative, regular products, and is probably applicable to others not mentioned here.

Two proofs are given for Theorem 4.8, the second of which explains and utilizes the method due to Magnus. Notations and conventions will be introduced there which are used in the proofs of Theorems 4.9 through 4.11.

Theorem 4.1. Let $A \circ B=A * B /(\mathfrak{R}[(A, A)], \mathfrak{R}[B])$. Then $\circ$ is a regular, noncommutative, nonassociative operation.

Proof. $\circ$ is regular because $(\mathfrak{N}[(A, A)], \mathfrak{N}[B]) \leqq(\mathfrak{R}[A], \mathfrak{N}[B])$ and G II; 1.2. Let $A$ be Abelian, and $B$ non-Abelian. Then $A \circ B=A * B$, while $\left(\left(b, b^{\prime}\right)\right.$, $a), b, b^{\prime} \in B, a \in A$ is a nontrivial element of $A * B$ which is mapped onto 1 in $B \circ A$. Thus $\circ$ is not commutative. Let $A, B$ be Abelian groups. Then $A \circ(B \circ C)=A * B * C$, while $((a, b), c), a \in A, b \in B, c \in C$ is a nontrivial element of $A * B * C$ mapped onto 1 in $(A \circ B) \circ C$. G II; 1.14 has been used. q.e.d.

The question arises, are all commutative, regular products associative? Here again, the answer is no.

Theorem 4.2. Let $A \circ B=A * B / \mathfrak{R}[((A, A),(B, B))]$; then $\circ$ is a commutative, nonassociative, regular operation.

Proof. $\circ$ is regular by G II; 1.2. It is commutative because of the symmetry of the kernel. Let $A$ be non-Abelian, $B$ and $C$ Abelian. Then $(A \circ B) \circ C$ $=A * B * C$. But $\left(\left(a, a^{\prime}\right),(b, c)\right), a, a^{\prime} \in A, b \in B, c \in C$ is a nontrivial element of $A * B * C$ which is mapped onto one in $A \circ(B \circ C)$. G II; 1.14 has again been used. q.e.d.

Comment. The same proof shows that $A \circ B=A * B /(\mathfrak{R}[(A, A)] \mathfrak{R},[(B, B)])$ is a commutative, nonassociative, regular product.

In the rest of this section more commutative, regular products will be defined and proved nonassociative. A summary of these products is given on p. 34 . 
Theorem 4.3. Let $A \circ B=A * B /((A, B),(A, B))$. Then $\circ$ is not associative.

Proof. Let $H=A \circ B, a \in A, b \in B, c \in C, h \in H$. Obviously, $u=((a, b)$, $(a, c))=1$ in $A \circ(B \circ C)$. It will be shown that $u \neq 1$ in $(A \circ B) \circ C$. $(A \circ B) \circ C=H * C /((H, C),(H, C))$. According to $\mathrm{G} \mathrm{II} ; 1.14,(H, C)$ is freely generated by $(h, c)$ for all $h \in H, c \in C$. By the definition of $H \circ C$, $(H, C)$ in $H \circ C$ is an Abelian group, the direct product of the infinite cyclic groups generated by $(h, c)$. Order the $(h, c)$ 's. Then every element of $(H, C)$ in $H \circ C$ can be uniquely expressed as a product of the form

$$
\begin{aligned}
\left(h_{1}, c_{1}\right)^{a_{1}}\left(h_{2}, c_{2}\right)^{a_{2}} \cdots\left(h_{n}, c_{n}\right)^{a_{n}}, \quad h_{i} \in H, c_{i} \in C, a_{i} \text { integers, } \\
\left(h_{i}, c_{i}\right) \neq\left(h_{j}, c_{j}\right) \text { if } i \neq j .
\end{aligned}
$$

All such expressions are distinct from 1 unless all factors equal 1. Now

$$
u=((a, b),(a, c))=((a, b), c)(a(a, b), c)^{-1}(a, c)
$$

as can be verified by applying $\mathrm{G} \mathrm{I} ; 2.1 .1$ and $\mathrm{G} \mathrm{I} ; 2.1 .3$ to $(c, a(a, b))$. Thus $u \neq 1$ in $H \circ C=(A \circ B) \circ C$. q.e.d.

Comment. Another variation of this proof is to consider $((H, C),(H, C))$ as a subgroup of $(H, C)$ in $H * C$. Using G I; 3.1, G I; 3.6 and G II; 1.14, $((H, C),(H, C))$ is generated by elements of the form

$$
\left(\left(h_{1}, c_{1}\right) \cdots\left(h_{k}, c_{k}\right),(h, c)\right), h_{i}, h \in H, c_{i}, c \in C .
$$

Thus in every element of $((H, C),(H, C))$ appears an even number of factors of the form $(h, c)$, since any cancellations will occur in pairs. But as shown above, the expansion of $u$ as an element of $(H, C)$ in $H * C$ has three factors, an odd number. The method using free associative rings which will be used later is also successful for proving Theorem 4.3. (See Theorem 4.10.)

Theorem 4.4. Let $A \circ B=A * B / P$, where $P=\mathfrak{N}\left[\left\{x^{2}, x \in(A, B)\right\}\right]$. Then $\circ$ is not associative.

Proof. Let $A=\{a\}, B=\{b\}, C=\{c\}$ and $a^{2}=b^{2}=c^{3}=1$. It will be shown that the order of $(A \circ B) \circ C$ is $2^{17} \cdot 3$, while the order of $A \circ(B \circ C)$ is $2^{27} \cdot 3$. By G II ; 1.2, the order of $U \circ V$ is the product of the orders of $U, V$ and $(U, V)$ in $U \circ V$. Accordingly, $A \circ B$ is of order 8 , since by $\mathrm{G} \mathrm{II} ; 1.14,(A, B)$ in $A * B$ is generated by $(a, b)$, and in $A \circ B,(a, b)^{2}=1$. In $(A \circ B) * C$, $(A \circ B, C)$ has $7 \times 2=14$ generators. A group in which every element is of order 2 is Abelian, since $x y x y=x y y x=1$ and hence $x y=y x$. Hence $(A \circ B, C)$ in $(A \circ B) \circ C$ is the direct product of 14 groups of order 2 . Thus the order of $(A \circ B) \circ C$ is $8 \cdot 3 \cdot 2^{14}=2^{17} \cdot 3$. Similarly, $B \circ C$ is of order $2 \cdot 3 \cdot 2^{2}=24$, and the order of $A \circ(B \circ C)$ is $2 \cdot 24 \cdot 2^{23}=2^{27} \cdot 3$. q.e.d.

Theorem 4.5. Let $A \circ B=A * B / P, P=\mathfrak{N}\left[\left\{(a, b)^{2}, a \in A, b \in B\right\}\right]$. Then $\circ$ is not associative. 
Proof. $(a b, c)(a b, c)=1$ in $(A \circ B) \circ C$, where $a \in A, b \in B, c \in C$. It will be shown that $(a b, c)(a b, c) \neq 1$ in $A \circ(B \circ C)$. Let $D=\mathrm{B} \circ C$. Then $(A, D)$ in $A * D$ is freely generated by $(a, d), a \in A, d \in D$, according to $\mathrm{G}$ II $; 1.14$. Thus $(A, D)$ in $A \circ D$ is the free product of cyclic groups of order two, each generated by $(a, d)$. By direct computation,

$$
(a b, c)(a b, c)=(b, a)(a, c b)(b(c, b), a)(a, c b(c, b))(b, c)^{2} \text { in } A * D .
$$

Since $(b, c)(b, c)=1$ and $(a, d)=(d, a)$ and $(b, c)=(c, b)$ in $A \circ D$, the above relation becomes

$$
(a b, c)(a b, c)=(a, b)(a, c b)(a, b(c, b))(a, b c) \text { in } A \circ D .
$$

But this is a nontrivial product in the free product $(A, D)$ in $A \circ D$. q.e.d.

Theorem 4.6. Let $A \circ B=A * B / P, G=A * B, P=\mathfrak{R}[\{(a b a b, g)$, for all $a \in A, b \in B, g \in G\}] \cap(A, B)$. Then $\circ$ is not associative.

Proof. To prove this theorem, we shall prove the following: (a) If $A$ is cyclic, and $B$ is the cyclic group of order 2 , then $(A, B)$ in $A \circ B$ is cyclic; in particular, let $A=\{a\}, B=\{b\}, a^{n}=b^{2}=1$; then $(a, b)^{k}=\left(a^{k}, b\right)$ and hence $(a, b)^{n}=1$. (b) If $A=\{r\}, B=\{s\}$, and $r^{4}=s^{3}=1$, then $A \circ B=A \times B$, i.e., $A \circ B$ degenerates into the direct product of $A$ and $B$.

In the proof of (a) and (b), we shall need the following identities which can be checked by direct computation: $(k, m, p$ are arbitrary integers)

(2) $\left(s^{k} \boldsymbol{r}^{m} s^{k} \boldsymbol{r}^{m}, \boldsymbol{r}^{p}\right)=\left(\boldsymbol{r}^{m}, s^{k}\right)\left(s^{k}, \boldsymbol{r}^{2 m}\right)\left(\boldsymbol{r}^{2 m}, s^{2 k}\right)\left(s^{2 k}, \boldsymbol{r}^{p+2 m}\right)\left(\boldsymbol{r}^{p+2 m}, s^{k}\right)\left(s^{k}, \boldsymbol{r}^{m+p}\right)$,

(3) $\quad\left(\boldsymbol{r}^{k} s^{m} \boldsymbol{r}^{k} s^{m}, \boldsymbol{r}^{p}\right)=\left(s^{m}, \boldsymbol{r}^{k}\right)\left(\boldsymbol{r}^{k}, s^{2 m}\right)\left(s^{2 m}, \boldsymbol{r}^{p+k}\right)\left(\boldsymbol{r}^{p+k}, s^{m}\right)\left(\boldsymbol{s}^{m}, \boldsymbol{r}^{p}\right)$,

(4) $\quad\left(s^{k} \boldsymbol{r}^{m} s^{k} \boldsymbol{r}^{m}, s^{p}\right)=\left(\boldsymbol{r}^{m}, s^{k}\right)\left(s^{k}, \boldsymbol{r}^{2 m}\right)\left(\boldsymbol{r}^{2 m}, s^{p+k}\right)\left(s^{p+k}, \boldsymbol{r}^{m}\right)\left(\boldsymbol{r}^{m}, s^{p}\right)$.

Let $b^{2}=1$; then

$$
\begin{aligned}
\left(a^{k} b a^{k} b, b\right) & =\left(b, a^{k}\right)\left(a^{2 k}, b\right)\left(b, a^{k}\right), \\
\left(b a^{m} b a^{m}, a^{p}\right) & =\left(a^{m}, b\right)\left(b, a^{2 m}\right)\left(a^{2 m+p}, b\right)\left(b, a^{m+p}\right), \\
\left(b a^{m} b a^{m}, b\right) & =\left(a^{m}, b\right)\left(b, a^{2 m}\right)\left(a^{m}, b\right), \\
\left(a^{m} b a^{m} b, a^{p}\right) & =\left(b, a^{m}\right)\left(a^{m+p}, b\right)\left(b, a^{p}\right) .
\end{aligned}
$$

Note that all the identities are variations of or derivable from (1) and (3).

Proof of (a). Using (8) and the definition of $A \circ B$, we have

$$
\left(b, a^{m}\right)\left(a^{m+p}, b\right)\left(b, a^{p}\right)=1 \text { in } A \circ B,
$$

i.e.,

$$
\left(a^{m+p}, b\right)=\left(a^{m}, b\right)\left(a^{p}, b\right) \text { for all integers } m, p .
$$

In particular, $\left(a^{2}, b\right)=(a, b)^{2}$ and, by induction, 


$$
\left(a^{m}, b\right)=\left(a^{m-1}, b\right)(a, b)=(a, b)^{m},
$$

and hence $1=\left(a^{n}, b\right)=(a, b)^{n}$. Inspection of identities (5), (6), (7), and (8), which are essentially the only identities holding in $A \circ B$, shows that $(a, b)^{k}$ $=1$ only if $n$ divides $k$. Since by G II $; 1.14,\left(a^{k}, b\right), k=1,2, \cdots, n-1$, generate $(A, B)$ in $A * B$, (a) is proved.

Proof of (b). In (3), let $p+k=4\left(r^{4}=1\right)$. Then using the definition of $A \circ B$

$$
\left(s^{m}, \boldsymbol{r}^{k}\right)\left(\boldsymbol{r}^{k}, s^{2 m}\right)\left(s^{m}, \boldsymbol{r}^{4-k}\right)=1 .
$$

Let $k=m=2$, then

$$
\left(r^{2}, s\right)=\left(r^{2}, s^{2}\right)^{2} .
$$

Let $m=k=1$, then $(s, r)\left(r, s^{2}\right)\left(s, r^{3}\right)=1$. Let $m=2, k=1$, then $\left(s^{2}, r\right)(r, s)\left(s^{2}, r^{3}\right)$ $=1$. From these two equalities in $A \circ B$, we obtain

$$
(r, s)=\left(r, s^{2}\right)\left(s, r^{3}\right)=\left(r, s^{2}\right)\left(r^{3}, s^{2}\right)
$$

or

$$
\left(s, r^{3}\right)=\left(r^{3}, s^{2}\right) .
$$

In (4) let $m=3, k=2, p=1$, then

$$
\left(r^{3}, s^{2}\right)\left(s^{2}, r^{2}\right)\left(r^{3}, s\right)=1 .
$$

Using (12) and (11),

$$
\left(s^{2}, r^{2}\right)=\left(s^{2}, r^{3}\right)\left(s, r^{3}\right)=1 .
$$

Using (9), this gives

$$
\left(r^{2}, s\right)=1 .
$$

In (2), let $m=1, p=2$, and use (13), (14) and the fact that 1 and 2 are essentially the only nontrivial values that $k$ can take. We obtain

$$
\left(\boldsymbol{r}, s^{k}\right)\left(s^{k}, r^{3}\right)=1 \text {. }
$$

Let $k=1,2$ :

$$
(r, s)=\left(r^{3}, s\right) ; \quad\left(r, s^{2}\right)=\left(r^{3}, s^{2}\right) .
$$

Combining with (11):

$$
\left(s, r^{3}\right)=(s, r)=\left(r^{3}, s^{2}\right)=\left(r, s^{2}\right) .
$$

In (2) let $k=p=1, m=2$, and use (14):

$$
\left(s^{2}, r\right)(r, s)\left(s, r^{3}\right)=1 \text {. }
$$

From (15) for $k=1$, and (17), it follows that

$$
\left(s^{2}, r\right)=1 \text {. }
$$


From (16), (18), (13), and (14), it follows that

$$
\left(r^{i}, s^{j}\right)=1, \quad i=1,2,3 ; \quad j=1,2 .
$$

With $\mathrm{G} \mathrm{II} ; 1.14$, this suffices to show that $(A, B)=1$ in $A \circ B$, which proves (b).

Proof of theorem. Let $A=\{a\}, B=\{b\}, C=\{c\}$ and $a^{4}=b^{3}=c^{2}=1$. Then by (b), $A \circ B=A \times B$, i.e., $A \circ B$ is the cyclic group of order 12 generated by $a b$. By (a), $(a b, c)$ generates $(A \circ B, C)$ in $(A \circ B) \circ C$, and $(a b, c)^{k}=\left((a b)^{k}, c\right)$. In particular, $(a b a b, c)=(a b, c)^{2} \neq 1$ in $(A \circ B) \circ C$. But $(a b a b, c)=1$ in $A \circ(B \circ C)$. q.e.d.

Theorem 4.7 is essentially a corollary to Theorem 4.6.

Theorem 4.7. Let $A \nabla B=A * B /((A, G),(B, G)) \cdot P$, where $G=A * B$, $P=\mathfrak{N}[\{(a b a b, g)$, for all $a \in A, b \in B, g \in G\}] \cap(A, B)$. Then $\nabla$ is not associative.

Proof. Let $A, B, C$ be the same groups as in the proof of Theorem 4.6. Then $D=A \nabla B=A \times B$, and $D$ is cyclic of order 12. Let $D * C=F$. Then by G I ; 3.5, $(D, F)=(D, D)(D, C)=(D, C)$ since $D$ is Abelian. Similarly $(C, F)$ $=(D, C)$. Thus $((D, F),(C, F))=1$ in $(A \nabla B) \nabla C$ reduces to $((D, C),(D, C))$ $=1$, i.e., $(D, C)$ is Abelian in $D \nabla C$. But from the proof of Theorem 4.6, $(d c d c, f)=1, d \in D, c \in C, f \in F$ is sufficient to make $(D, C)$ in $D \nabla C$ Abelian. Hence $(A \nabla B) \nabla C=(A \circ B) \circ C$, where $\circ$ refers to the $\circ$ of Theorem 4.6, and thus in $(A \nabla B) \nabla C,(a b a b, c) \neq 1$. But $(a b a b, c)=1$ in $A \nabla(B \nabla C)$. q.e.d.

Comment. In $\S 5$, it will be shown that $\Psi$ is associative, where $A \Psi B$ $=(A * B) /((A, G),(B, G)), G=A * B$ (Theorem 5.1 , for $m=n=1$ ). It was conjectured that by multiplying the "nonassociative factor" $P=\{(a b a b, g)\}$ by the "associative factor" $((A, G),(B, G))$, an associative product might be produced. Theorem 4.7 shows that this conjecture was mistaken.

Theorem 4.8. Let $A \circ B=A * B /((A, B),(G, G)), G=A * B$. Then $\circ$ is not associative.

Proof. Two proofs will be given here. The first will be a special proof designed for just this $\circ$. The second will be a proof, based on the work of Magnus given in $\S 3$. This second proof is a general method which will be used to show a large number of products are nonassociative. Both methods are given here to indicate the superiority of the second method over the first.

First proof. Let $A * B * C=F$. Then by the second theorem of isomorphism

$$
\begin{aligned}
& A \circ(B \circ C) \cong A * B * C / \mathfrak{N}[((B, C),(B * C, B * C))] \cdot((B * C, A),(F, F))=F / Q, \\
& (A \circ B) \circ C \cong A * B * C / \mathfrak{N}[((A, B),(A * B, A * B))] \cdot((A * B, C),(F, F))=F / P .
\end{aligned}
$$

Suppose that $\circ$ is associative. Then $Q=P$. Let $a, a^{\prime} \in A, b, b^{\prime} \in B, c, c^{\prime} \in C$. Obviously $\left((c, b),\left(a, a^{\prime}\right)\right) \in P$. Hence $\left((c, b),\left(a, a^{\prime}\right)\right) \in Q$. From considerations of symmetry it follows that $\left(\left(b, b^{\prime}\right),\left(a, a^{\prime}\right)\right) \in Q$. Hence $\left(\left(b, b^{\prime}\right),\left(a, a^{\prime}\right)\right) \in P$. Let $D=A \circ B$. Then $\left(\left(b, b^{\prime}\right),\left(a, a^{\prime}\right)\right)=1 \bmod ((D, C),(D * C, D * C))$ in $D * C$. 
Since $\left(\left(b, b^{\prime}\right),\left(a, a^{\prime}\right)\right) \in D$ and by G II $; 1.2$ every element of $D * C$ can be uniquely expressed as $d c u, d \in D, c \in C, u \in(D, C),\left(\left(b, b^{\prime}\right),\left(a, a^{\prime}\right)\right)=1$ in $D$. Thus if we can find two groups $A$ and $B$ such that $\left(\left(b, b^{\prime}\right),\left(a, a^{\prime}\right)\right) \neq 1$ in $A \circ B$, we have proved the nonassociativity of $\circ$. Let $M=\{a, b\} ; N=\{c, d\}$; $((a, b), a)=((a, b), b)=a^{2}=b^{2}=1 ;((c, d), c)=((c, d), d)=c^{2}=d^{2}=1$. Then $((a, b),(c, d)) \neq 1$ in $M \circ N$. When this is proved, the nonassociativity of $\circ$ is proved: $M$ and $N$ are both non-Abelian groups of order 8 . (Note that $((a, b)$, $a)=(b, a)(b, a)=1$. $) M \cong N$. The elements of $M$ are $a, b, a b, b a,(a, b), b a b$, $a b a$, and 1. All elements are of order 2 except $a b$ and $b a$ which are of order 4 . Similar statements hold for $N$.

By Theorem 3.5, and G I; 4.3 and $\mathrm{G} \mathrm{I} ; 3.1,((M, N),(M * N, M * N))$ $=((M, N),(M, N))((M, N), \mathfrak{R}[(M, M)])((M, N), \mathfrak{R}[(N, N)]) .((M, N)$, $(M, N))=1$ in $M \circ N$ means that $(M, N)$ is Abelian in $M \circ N$. Since $(M, N)$ is finitely generated, this means that $(M, N)$ in $M \circ N$ is the direct product of cyclic groups. $(a, b)$ is the only nontrivial element of $(M, M)$. From $((M, N)$, $(M, M))=1$, we obtain (let $m \in M, n \in N$ )

$$
((m, n),(a, b))=(n, m)(m(a, b), n)(n,(a, b))=1
$$

or

$$
(m(a, b), n)=(m, n)((a, b), n) \quad \text { for all } m \in M, n \in N .
$$

Similarly from $((M, N),(N, N))=1$, we obtain

$$
(n(c, d), m)=(n, m)((c, d), m) \quad \text { for all } m \in M, n \in N .
$$

It turns out that these are essentially the only relations that hold in $(M, N)$ in $M \circ N$. For example,

$$
\left(\left(m, n_{1}\right), n^{-1}(a, b) n\right)=1 \text { in } M \circ N, n, n_{1} \in N, m \in M,
$$

and this gives

$$
\begin{array}{rr}
\left(\left(m, n_{1}\right),(a, b)((a, b), n)\right) & \text { (using G I; 2.1.3) } \\
=\left(\left(m, n_{1}\right),((a, b), n)\right)(n,(a, b))\left(\left(m, n_{1}\right),(a, b)\right)((a, b), n)=1
\end{array}
$$

which gives no new relations. The complete verification consists of more computations of the type just described.

The chart on p. 439 gives the results of (1) and (2). It turns out that $(M, N)$ is the direct product of nine infinite cyclic groups and seven cyclic groups of order 2. The elements of order 2 are of the form

$$
((a, b), n) \text { and } \quad((c, d), m) \text {. }
$$

In particular, $((a, b),(c, d)) \neq 1$. q.e.d.

Second proof. Let $P$ and $Q$ be the same as in the first proof. We shall show that $\left((a, b),\left(c, c^{\prime}\right)\right) \notin P$, where $a \in A, b \in B, c, c^{\prime} \in C$. Obviously $((a, b)$, $\left.\left(c, c^{\prime}\right)\right) \in Q$. We shall use Theorem MI proved by W. Magnus as stated in $\S 3$. 


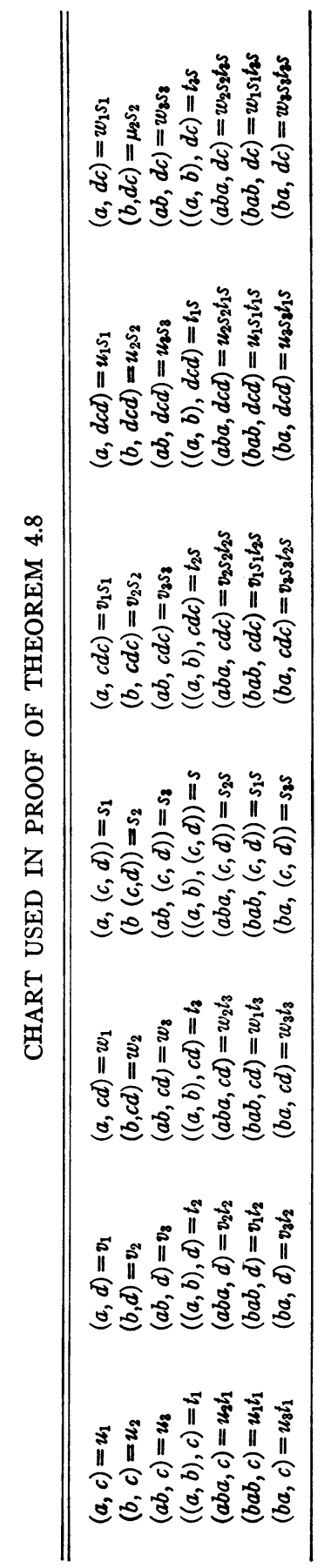


Let $x, y, z_{1}, z_{2}$ be generators of a free associative ring $R$ over the integers. Let $a=1+x, b=1+y, c_{i}=1+z_{i}$ as indicated in Theorem MI, i.e., we shall assume that $A, B$ are infinite cyclic groups generated by $a$ and $b$ respectively and that $C=\left\{c_{1}, c_{2}\right\}$ is the free product of two infinite cyclic groups. We shall show that $\left((a, b),\left(c_{1}, c_{2}\right)\right)$ corresponds to an element which cannot occur in $P$. Since our argument will depend only on the literal coefficients of the elements of $R$, we shall not write the numerical coefficients. For example, instead of writing a typical element of $A$ as

$$
1+n_{1} x+n_{2} x^{2}+\cdots,
$$

$n_{i}$ integers,

we shall write a typical element of $A$ as

$$
1+x+x^{2}+\cdots .
$$

We first compute the literal coefficients of a typical element of $P$. We shall use Theorem 3.8, and it will be sufficient to consider only the first nontrivial term of each series.

$P$ is the product of $\mathfrak{R}[((A, B),(A * B, A * B))]$ and $((A * B, C),(F, F))$, where $F=A * B * C$. We shall show that elements of the first factor considered as elements of $R$ start with polynomials of degree 5 , while those of $((A * B, C)$, $(F, F))$ start with polynomials of degree 4 . Thus if we can show that there are terms in the fourth degree polynomial with which $\left((a, b),\left(c, c^{\prime}\right)\right)$ starts which do not appear in any fourth degree polynomial corresponding to an element of $((A * B, C),(F, F))$, we have proved the nonassociativity of our product.

$\mathfrak{N}[((A, B),(A * B, A * B))]$ : Let a typical element of $A$ be $1+x+\cdots$ and similarly a typical element of $B$ be $1+y+\cdots$ and a typical element of $A * B$ be $1+x+y+\cdots$. Then using Theorem 3.8(a) and the fact that $[u, u]=0$ for any $u$, we have

$$
1+[x, y]+\cdots \text { as a typical element of }(A, B),
$$

and

$$
1+[x, y]+\cdots \text { as a typical element of }(A * B, A * B) .
$$

Since $[[x, y],[x, y]]=0$, elements of $((A, B),(A * B, A * B))$ start with a polynomial of degree 5 . (Here we have used Theorem 3.8(d).) Use of Theorem 3.8(c) shows that $\mathfrak{R}[((A, B),(A * B, A * B))]$ starts with elements of degree 5 .

$((A * B, C),(F, F))$ : Let typical elements of $A * B, C$, and $F$ be $1+x+y$ $+\cdots, 1+z_{1}+z_{2}+\cdots$ and $1+x+y+z_{1}+z_{2}+\cdots$ respectively. Then typical elements of $(A * B, C)$ and $(F, F)$ are

$$
1+\left[x, z_{1}\right]+\left[x, z_{2}\right]+\left[y, z_{1}\right]+\left[y, z_{2}\right]+\cdots
$$

and

$$
1+\left[x, z_{1}\right]+\left[x, z_{2}\right]+\left[y, z_{1}\right]+\left[y, z_{2}\right]+[x, y]+\left[z_{1}, z_{2}\right]+\cdots
$$


respectively. Then a typical element from $((A * B, C),(F, F))$ is

$$
1+\left[\left[x, z_{1}\right],\left[x, z_{2}\right]\right]+\left[\left[x, z_{i}\right],\left[y, z_{i}\right]\right]
$$

$$
\begin{aligned}
& +\left[\left[y, z_{1}\right],\left[y, z_{2}\right]\right]+\left[\left[x, z_{i}\right],[x, y]\right]+\left[\left[x, z_{i}\right],\left[z_{1}, z_{2}\right]\right] \\
& +\left[\left[y, z_{i}\right],[x, y]\right]+\left[\left[y, z_{i}\right],\left[z_{1}, z_{2}\right]\right]+\cdots
\end{aligned}
$$

where by $\left[x, z_{i}\right]$ and $\left[y, z_{i}\right]$ is meant summation over both values of $i(i=1,2)$. For the sake of brevity, we can write (1) as

$$
1+[[\alpha, \beta],[\gamma, \delta]]+\cdots
$$

where $\alpha=x$ or $y, \beta=z_{1}$ or $z_{2}$, and $\gamma \neq \delta$, and summation is assumed over all $\alpha, \beta, \gamma, \delta$ which satisfy these conditions.

$$
\begin{gathered}
\left((a, b),\left(c_{1}, c_{2}\right)\right): a=1+x+\cdots, \quad b=1+y+\cdots, \quad c_{i}=1+z_{i}+\cdots, \\
(a, b)=1+[x, y]+\cdots, \quad\left(c_{1}, c_{2}\right)=1+\left[z_{1}, z_{2}\right]+\cdots,
\end{gathered}
$$

and

$$
\left((a, b),\left(c_{1}, c_{2}\right)\right)=1+\left[[x, y],\left[z_{1}, z_{2}\right]\right]+\cdots .
$$

Consider $x y z_{1} z_{2}$ which appears in $\left((a, b),\left(c_{1}, c_{2}\right)\right)$, and compare it with the fourth degree polynomial represented in (2). The only terms in which $z_{1} z_{2}$ appears as the last two factors of a fourth degree term, $x y$ does not appear with it. Remember that elements of a free associative ring do not commute, so that

$$
\left[\left[x, z_{1}\right],\left[y, z_{2}\right]\right]=\left(x z_{1}-z_{1} x\right)\left(y z_{2}-z_{2} y\right)-\left(y z_{2}-z_{2} y\right)\left(x z_{1}-z_{1} x\right)
$$

has no terms of the form $x y z_{1} z_{2}$. q.e.d.

Theorem 4.9. Let $A \circ B=A * B /((\mathfrak{R}[A] ; \mathfrak{R}[A]),(\mathfrak{R}[B], \mathfrak{N}[B]))$. Then $\circ$ is not associative.

Proof. The method of free associative rings used in the proof of Theorem 4.8 is used again. By the second law of isomorphism,

$$
\begin{array}{r}
\left.(A \circ B) \circ C \cong A * B * C / \mathfrak{N}\left[\left(\mathfrak{N}^{G}[A], \mathfrak{R}^{G}[A],\right)\left(\mathfrak{R}^{G}[B], \mathfrak{R}^{G}[B]\right)\right)\right] \\
\cdot((\mathfrak{N}[A * B], \mathfrak{N}[A * B]),(\mathfrak{N}[C], \mathfrak{N}[C]))=F / P
\end{array}
$$

and

$$
\begin{array}{r}
A \circ(B \circ C) \cong A * B * C / \mathfrak{N}\left[\left(\left(\mathfrak{N}^{E}[B], \mathfrak{N}^{E}[B]\right),\left(\mathfrak{N}^{E}[C], \mathfrak{N}^{E}[C]\right)\right)\right] \\
\cdot((\mathfrak{N}[B * C], \mathfrak{N}[B * C]),[\mathfrak{N}[A], \mathfrak{N}[A]))=F / Q,
\end{array}
$$

where $F=A * B * C, G=A * B, E=B * C$. Let $A=\left\{a_{1}, a_{2}\right\}, B=\{b\}, C=\{c\}$, $A$ being the free product of two infinite cyclic groups, and $B$ and $C$ being infinite cyclic groups. Using the method of free associative rings, we shall show that $P$ is made up of elements which start with polynomials of degree 5 , i.e., 
using Theorem MI, $P \leqq Z_{5}$, while $\left((b, c),\left(a_{1}, a_{2}\right)\right)$ starts with a polynomial of degree four, i.e., $Q \cap Z_{4} \neq 0$, and hence $P \neq Q$.

Using the same notation and conventions as in Theorem 4.8 , let $a_{i}=1+x_{i}$, $b=1+y, c=1+z$. Then from Theorem 3.8(c), it follows that typical elements from $\mathfrak{N}[A], \mathfrak{N}[B]$, and $\mathfrak{N}[C]$ are of the form $1+x_{1}+x_{2}+\cdots, 1+y+\cdots$, and $1+z+\cdots$, respectively. Using Theorem $3.8(\mathrm{a})$, elements from $\left(\mathfrak{R}^{G}[B], \mathfrak{N}^{G}[B]\right)$ and $(\mathfrak{R}[C], \mathfrak{N}[C])$ start with polynomials of at least the third degree. (Remember that $[u, u]=0$ for any $u$.) Elements from $(\mathfrak{N}[A], \mathfrak{N}[A])$ and $\left(\mathfrak{N}^{G}[A], \mathfrak{N}^{a}[A]\right)$ start with $1+\left[x_{1}, x_{2}\right]+\cdots$. Elements from $(\mathfrak{N}[A * B], \mathfrak{N}[A * B])$ start with polynomials of the second degree. Thus, using Theorem 3.8, all elements from $P$ start with polynomials of the fifth degree. But $\left((b, c),\left(a_{1}, a_{2}\right)\right)$ starts with an expansion of the form

$$
1+\left[[y, z],\left[x_{1}, x_{2}\right]\right]+\cdots
$$

whose first polynomial is of the fourth degree. q.e.d.

TheOREM 4.10. Let $A \circ B=A * B / k((A, B),(A, B)), k$ any non-negative integer. Then $\circ$ is not associative.

Proof. The method of free associative rings is used. Let $A, B, C$ be infinite cyclic groups, $A=\{a\}, B=\{b\}, C=\{c\}, a=1+x, b=1+y, c=1+z$. As before,

$$
(A \circ B) \circ C \cong A * B * C / \mathfrak{N}\left[{ }_{k}((A, B),(A, B))_{G}\right] \cdot{ }_{k}((A * B, C),(A * B, C))=F / P
$$

and

$$
A \circ(B \circ C) \cong A * B * C / \mathfrak{N}\left[{ }_{k}((B, C),(B, C))_{E}\right] \cdot{ }_{k}((B * C, A),(B * C, A))=F / Q,
$$

where $F=A * B * C, G=A * B, E=B * C$. Now

$$
(\cdots((((a, c),(b, c)), c), c), \cdots, c)
$$

obviously $\in P$. We shall show that it is not in $Q$.

$\mathfrak{N}\left[{ }_{k}((B, C),(B, C))_{E}\right]$ : Using previous notation and conventions, typical elements from $(B, C)$ have the form $1+[y, z]+\cdots$. Hence elements from $((B, C),(B, C))$ start with polynomials of degree 5 , and elements from ${ }_{k}((B, C),(B, C))$ start with elements of degree $k+5$.

${ }_{k}((B * C, A),(B * C, A))$ : Elements from $B * C$ start with polynomials of the form $1+y+z+\cdots$, and elements from $(B * C, A)$ start with polynomials of the form $1+[y, x]+[z, x]+\cdots$. Thus elements from $((B * C, A)$, $(B * C, A))$ start with elements of the form $1+[[y, x],[z, x]]+\cdots$ i.e., all polynomials of degree 4 have at least two $x$ 's in them. Thus all polynomials of degree $k+4$ in ${ }_{k}((B * C, A),(B * C, A))$ have at least two $x$ 's in them.

$(\cdots((((a, c),(b, c)), c), c), \cdots, c):(a, c)$ and $(b, c)$ have the form $1+[x, z]+\cdots$ and $1+[y, z]+\cdots$ respectively. Hence $((a, c),(b, c))$ has the form $1+[[x, z],[y, z]]+\cdots$, i.e., there is only one $x$ in its polynomials 
of degree 4. This proves the theorem for $k=0$. For $k \geqq 1,(\cdots((((a, c)$, $(b, c)), c), c), \cdots, c)$ has the expansion

$$
1+[\cdots[[[[x, z],[y, z]], z], z], \cdots, z]+\cdots
$$

which again has only one $x$ in its polynomials of degree $k+4$.

Theorem 4.11. Let $A \circ B=A * B / k((A, G),(B, G)), G=A * B, k \geqq 1$. Then $\circ$ is not associative.

Note. In $\S 5$, it will be shown that this product is associative for $k=0$. Therefore, the assumption that $k \geqq 1$ is essential.

Proof. The method of free associative rings is used. Let $A=\left\{a_{1}, a_{2}\right\}$, $B=\{b\}, C=\{c\}, a_{i}=1+x_{i}, b=1+y, c=1+z$, i.e., $A$ is the free product of two infinite cyclic groups and $B$ and $C$ are infinite cyclic. Then $F=A * B * C$, $G=A * B$, and $E=B * C,(A \circ B) \circ C \cong A * B * C / P$, where $P=\mathfrak{N}\left[{ }_{k}((A, G)\right.$, $\left.(B, G))_{G}\right] \cdot{ }_{k}((A * B, F),(C, F)), A \circ(B \circ C) \cong F / Q$, where $Q=\mathfrak{N}\left[{ }_{k}((B, E)\right.$, $\left.(C, E))_{E}\right] \cdot{ }_{k}((B * C, F),(A, F)) . u=\left(\cdots\left(\left(\left(a_{1}, b\right),\left(a_{2}, b\right)\right), c\right), \cdots, c\right)$ obviously is in $Q$. We show that it is not in $P$ :

$\mathfrak{N}\left[{ }_{k}((A, G),(B, G))_{G}\right]$ : Elements from this group are of the form

$$
1+f\left(x_{i}, y\right)+\cdots
$$

where $f\left(x_{i}, y\right)$ is a polynomial of degree $k+4$ in the $x_{i}$ and $y$. As usual, Theorem 3.8 has been used here.

${ }_{k}((A * B, F),(C, F))$ : Elements from $G, C$, and $F$ are of the form $1+x_{1}+x_{2}+y+\cdots, 1+z+\cdots$, and $1+x_{1}+x_{2}+y+z+\cdots$ respectively. $(A * B, F)$ is of the form $1+[\alpha, \beta]+\cdots$ where $\alpha \neq \beta ; \alpha$ or $\beta=x_{1}, x_{2}$, or $y$; and summation is assumed over all $\alpha, \beta$ satisfying these conditions. Elements from $(C, F)$ are of the form $1+[\gamma, \delta]+\cdots$ where $\gamma \neq \delta ; \gamma$ or $\delta=z$; and summation is assumed over all $\gamma, \delta$ satisfying these conditions! Thus elements from $((A * B, F),(C, F))$ are of the form

$1+[[\alpha, \beta],[\gamma, \delta]]+\cdots$, where $\alpha, \beta, \gamma, \delta$ satisfy the conditions stated above and summation is assumed over all $\alpha, \beta, \gamma, \delta$ satisfying these conditions. Elements from ${ }_{k}((A * B, F),(C, F))$ are of the form

$$
1+\left[\cdots\left[[[\alpha, \beta],[\gamma, \delta]], \epsilon_{1}\right], \cdots, \epsilon_{k}\right]+\cdots
$$

where $\alpha, \beta, \gamma, \delta$ are as above, and $\epsilon_{j}=x_{i}, y$, or $z$ and summation is assumed over all $\alpha, \beta, \gamma, \delta, \epsilon_{i}$ satisfying these conditions.

$u:\left(\left(a_{1}, b\right),\left(a_{2}, b\right)\right)$ is of the form

$$
1+\left[\left[x_{1}, y\right],\left[x_{2}, y\right]\right]+\cdots
$$

hence $u$ is of the form

$$
1+\left[\cdots\left[\left[\left[\left[x_{1}, y\right],\left[x_{2}, y\right]\right], z\right], z\right], \cdots, z\right] .
$$

Consider $x_{1} y y x_{2} z^{k}$ which appears in the expansion of $u$. We show that it 
does not appear in any term of the form (2). In every term of (2) at least one of the following appears as a factor: $\alpha \beta \gamma \delta, \quad \beta \alpha \gamma \delta, \quad \alpha \beta \delta \gamma, \quad \beta \alpha \delta \gamma, \quad \gamma \delta \alpha \beta, \quad \gamma \delta \beta \alpha, \quad \delta \gamma \alpha \beta, \quad \delta \gamma \beta \alpha$.

Now consider $\left(x_{1} y y x_{2}\right) z^{k}, x_{1}\left(y y x_{2} z\right) z^{k-1}, x_{1} y\left(y x_{2} z z\right) z^{k-2}, x_{1} y y\left(x_{2} z z z\right) z^{k-3}, \cdots$. Consider the sets of four factors in the parenthesis of each of these terms. If $x_{1} y y x_{2} z^{k}$ appears in (2), at least one of the sets of four factors must be of the form (3). $x_{1} y y x_{2}$ cannot be one of the terms of (3) because all terms of (3) contain at least one $z$. $y y x_{2} z$ cannot be one of the terms of (3) because $y y$ never appears in (3) as the first two factors since $\alpha \neq \beta$ and $\gamma \neq \delta$. Similarly $y x_{2} z z$ cannot be one of the terms of (3). For the same reason, all other possibilities are excluded. Hence $x_{1} y y x_{2} z^{k}$ never appears as one of the terms of (2). $x_{1} y y x_{2} z^{k}$ does not appear in any term of (1) because of the presence of $z^{k}$. Here is where the assumption $k \geqq 1$ is used. q.e.d.

5. Associative products. In this section, a denumerable number of commutative, regular products will be defined and proved associative. It will be shown that these products include products that are different from Golovin's nilpotent products, and that nilpotent products form a special case of these products. In Theorem 5.1 these products are defined and proved associative. The heart of the proof lies in Theorem 3.3. The rest of the theorems of this section "tie up odds and ends," and Theorem 5.5 generalizes the product of Theorem 5.1.

Theorem 5.1. Let $A \circ B=A * B /\left({ }_{m} A,{ }_{n} B\right)\left({ }_{n} A,{ }_{m} B\right)$, where $m$, $n$ are any two non-negative integers. Then $O$ is a fully regular product.

Proof. That $\circ$ is regular follows from G II; 1.2 and the fact that $\left({ }_{m} A,{ }_{n} B\right)$ . $\left({ }_{n} A,{ }_{m} B\right) \leqq(A, B)$. ○ is commutative since $\left({ }_{m} A,{ }_{n} B\right)\left({ }_{n} A,{ }_{m} B\right)$ is symmetric with respect to $A$ and $B$. The only problem is the associativity. Let $F=A * B * C, G=A * B, E=B * C$, then

$(A \circ B) \circ C \cong F / P, \quad P=\mathfrak{N}\left[\left({ }_{m} A_{G},{ }_{n} B_{G}\right)\right] \mathfrak{N}\left[\left({ }_{n} A_{G},{ }_{m} B_{G}\right)\right] \cdot\left({ }_{m} A * B,{ }_{n} C\right)\left({ }_{n} A * B,{ }_{m} C\right)$

and

$A \circ(B \circ C) \cong F / Q, \quad Q=\mathfrak{N}\left[\left({ }_{m} B_{E},{ }_{n} C_{E}\right)\right] \mathfrak{\Re}\left[\left({ }_{n} B_{E},{ }_{m} C_{E}\right)\right] \cdot\left({ }_{m} B * C,{ }_{n} A\right)\left({ }_{n} B * C,{ }_{m} A\right)$.

We want to show that $P=Q$. We shall show that $Q \leqq P$. By symmetry, the opposite inequality will follow, and hence the equality. $\mathfrak{N}\left[\left({ }_{m} B_{E},{ }_{n} C_{E}\right)\right]$ - $\mathfrak{R}\left[\left({ }_{n} B_{E},{ }_{m} C_{E}\right)\right] \leqq P$ by G I; 2.4 .2 , and the normality of members of a lower central series. Using G I; 5.3 , G I ; 4.3, we obtain

$$
\left({ }_{m} B * C,{ }_{n} A\right)=\left({ }_{m} B \cdot{ }_{m} C,{ }_{n} A\right)=\left({ }_{m} B,{ }_{n} A\right)\left({ }_{m} C,{ }_{n} A\right),
$$

and similarly

$$
\left({ }_{n} B * C,{ }_{m} A\right)=\left({ }_{n} B,{ }_{m} A\right)\left({ }_{n} C,{ }_{m} A\right) .
$$


Obviously $\left({ }_{m} C,{ }_{n} A\right)\left({ }_{n} C,{ }_{m} A\right) \leqq P$. The problem is to show that $\left({ }_{m} B,{ }_{n} A\right)$ $\cdot\left({ }_{n} B,{ }_{m} A\right) \leqq P$. By Theorem 3.3

$$
{ }_{k} B \leqq{ }_{k} B_{G}\left({ }_{k} G \cap{ }_{k} C\right)
$$

and similarly for ${ }_{k} A$. Hence, if we let ${ }_{k} G \cap_{k} C=N_{k}$ and use Theorem 3.3 and Theorem 3.2,

$$
\begin{aligned}
\left({ }_{m} B,{ }_{n} A\right) & \leqq\left({ }_{m} B_{G} \cdot N_{m},{ }_{n} A_{G} \cdot N_{n}\right) \\
& \leqq \mathfrak{N}\left[\left({ }_{m} B_{G},{ }_{n} A_{G}\right)\left({ }_{m} B_{G},{ }_{n} C\right)\left({ }_{m} C,{ }_{n} A_{G}\right)\left({ }_{m} C,{ }_{n} G\right)\right] \leqq P .
\end{aligned}
$$

Similarly $\left({ }_{n} B,{ }_{m} A\right) \leqq P$. q.e.d.

Theorem 5.2. If $m=0$, and $n=k$, then $A \circ B$ in Theorem 5.1 is Golovin's kth nilpotent product.

Proof. Theorem 5.2 follows immediately from Theorem 3.7 and the definition of nilpotent product.

Theorem 5.3. If neither $m$ nor $n$ are $=0$ in Theorem 5.1 , then $A \circ B$ is not a nilpotent product.

Proof. By G II ; 6.3, if $A$ and $B$ are finite, then $A(k) B$ is finite. Let $A=\{a\}$, $B=\{b\}, a^{2}=b^{2}=1$. We shall show that $A \circ B=A * B$ in this case, and thus is infinite. Hence, it cannot be nilpotent. By G II; $1.14(A, B)$ in $A * B$ $=\{(a, b)\}$, i.e., $(A, B)$ is infinite cyclic, and hence Abelian. Thus $((A, B)$, $(A, B))=1$. Now using G I ; $3.5,{ }_{1} A=(A, G)=(A, B)(A, A)=(A, B)$. Since the lower central series is a decreasing series, ${ }_{k} A \leqq(A, B)$ for $k \geqq 1$. Similarly ${ }_{k} B \leqq(A, B)$ for $k \geqq 1$. Thus $\left({ }_{m} A,{ }_{n} B\right) \leqq((A, B),(A, B))=1$ if $m, n \geqq 1$. But then $A \circ B=A * B$, if $m, n \geqq 1$. q.e.d.

Theorem 5.4. Let $A \circ B=A * B /\left({ }_{m} A,{ }_{n} B\right)\left({ }_{n} A,{ }_{m} B\right)$. Then

$$
A_{1} \circ A_{2} \circ \cdots \circ A_{k} \cong \prod_{i=1}^{k} A_{i} / \prod_{i, j ; i \neq j}^{k}\left({ }_{m} A_{i},{ }_{n} A_{j}\right) .
$$

Proof. Let $k=3$. In the proof of Theorem 5.1, we showed that $P \geqq\left({ }_{m} A,{ }_{n} C\right)$ $\cdot\left({ }_{m} B,{ }_{n} C\right)\left({ }_{m} A,{ }_{n} B\right)\left({ }_{n} A,{ }_{m} C\right)\left({ }_{n} B,{ }_{m} C\right)\left({ }_{n} A,{ }_{m} B\right)$. By G I; 5.3 the inequality also holds the other way, and hence is an equality. Since $P=Q$ (Theorem 5.1), Theorem 5.4 is proved for $k=3$. For $k>3$, use induction and a proof similar to that of Theorem 5.1. Let

THEOREM 5.5. Let $m_{1}, \cdots, m_{k}, n_{1}, n_{2}, \cdots, n_{k}$ be $2 k$ non-negative integers.

$$
A \circ B=A * B / \prod_{i=1}^{k}\left({ }_{m_{i}} A,{ }_{n_{i}} B\right)\left({ }_{n_{i}} A,{ }_{m_{i}} B\right) .
$$

Then $\circ$ is associative. If all the $m_{i}, n_{i} \geqq 1$, then $\circ$ is not nilpotent. 
Proof. The proof of the associativity is the same as that for Theorem 5.1. The proof of non-nilpotentcy is the same as that for Theorem 5.3.

6. MacLane's postulate. In this section MacLane's postulate will be defined and it will be shown that all the commutative, regular products mentioned so far satisfy this postulate. Then the relation between S. Moran's verbal products and those of $\$ 5$ will be briefly summarized. Finally a denumerable number of fully regular products which do not satisfy MacLane's postulate will be given. This example shows that MacLane's postulate is independent of the other postulates.

Definition. Let $A \circ B$ be a product of $A$ and $B$; let $M$ and $N$ be normal subgroups of $A$ and $B$ respectively. Then o satisfies MacLane's postulate if

$$
\frac{A \circ B}{\mathfrak{N}[M N]} \cong \frac{A}{M} \circ \frac{B}{N}
$$

under the obvious mapping for all $A$ and $B$.

Comment. If a product satisfies MacLane's postulate, then the formation of the product is to some extent independent of the structure of $A$ and $B$; this will be shown clearly in the example of Theorem 6.3.

THEOREM 6.1. The nonassociative products of Theorems 4.2 through 4.11, and the associative products of Theorems 5.1 and 5.5 all satisfy MacLane's postulate; i.e., all the commutative, regular products mentioned so far satisfy MacLane's postulate.

Proof. By the second law of isomorphism:

$$
\frac{A \circ B}{\mathfrak{N}[M N]} \cong \frac{A * B}{\mathfrak{N}[M N] P}
$$

for some subgroup $P$ of $A * B$.

$$
\frac{A}{M} \circ \frac{B}{N} \cong \frac{\frac{A}{M} * \frac{B}{N}}{P^{\prime}} \cong \frac{A * B}{\mathfrak{R}[M N] P^{\prime \prime}}
$$

for some subgroup $P^{\prime}$ of

$$
\frac{A}{M} * \frac{B}{N}
$$

and some subgroup $P^{\prime \prime}$ of $A * B$. For each of the products mentioned in the statement of the theorem, compute the corresponding $P$ and $P^{\prime \prime}$. If they are equal, then MacLane's postulate holds; if not, it does not. A computation will show that it does. q.e.d.

In [5], S. Moran has defined verbal products and shown that they are 
fully regular. The following theorem shows that the products of Theorem 5.1 (and hence those of Theorem 5.5) are special cases of verbal products.

Theorem 6.2. Let $G=A * B$. Then $\left({ }^{m} G,{ }^{n} G\right) \cap(A, B)=\left({ }_{m} A,{ }_{n} B\right)\left({ }_{n} A,{ }_{m} B\right)$, where $m, n$ are two arbitrary, fixed non-negative integers.

Proof. For the proof of this theorem, the following lemmas will be useful:

Lemma 1. $(p q, s t)=(p, t)((p, t), q)(q, t)(t,(s, p q))(p, s)((p, s), q)(q, s)$. This identity can be verified directly, or G I; 2.1 .2 and $\mathrm{G} \mathrm{I} ; 2.1 .3$ can be applied several times.

Lemma 2. Let $G=A * B$ and $g_{i}=a_{i} b_{i} u_{i}, a_{i} \in A, b_{i} \in B, u_{i} \in(A, B)$. (Use is made here of the unique representation theorem, G II; 1.2.) Let

$$
\begin{aligned}
{ }^{k} g & =\left(\left(\cdots\left(\left(g_{0}, g_{1}\right), g_{2}\right), \cdots\right), g_{k}\right), \\
{ }^{k} a & =\left(\left(\cdots\left(\left(a_{0}, a_{1}\right), a_{2}\right), \cdots\right), a_{k}\right), \\
{ }^{k} b & =\left(\left(\cdots\left(\left(b_{0}, b_{1}\right), b_{2}\right), \cdots\right), b_{k}\right) ;
\end{aligned}
$$

then

$$
{ }^{k} a={ }^{k} a^{k} b v, \quad v \in{ }_{k-1}(A, B), \quad \text { for } k=1,2, \cdots .
$$

Proof of Lemma 2. Proof is by induction on $k$.

$k=1$ : We want to show that $\left(a_{0} b_{0} u_{0}, a_{1} b_{1} u_{1}\right)=\left(a_{0}, a_{1}\right)\left(b_{0}, b_{1}\right) u, u \in(A, B)$. First, use Lemma 1, on

$$
\left(a_{0} b_{0}, a_{1} b_{1}\right) \text {. }
$$

Using the fact that $(A, B)$ is normal in $A * B$ (and hence that $(w, g) \in(A, B)$ for all $w \in(A, B)$ and $g \in G)$, Theorem $3.7\left(\left({ }_{m} A,{ }_{n} B\right) \leqq{ }_{m+n}(A, B)\right)$, and the fact that every lower central series is a decreasing sequence, we obtain

$$
\left(a_{0} b_{0}, a_{1} b_{1}\right)=\left(b_{0}, b_{1}\right)\left(a_{0}, a_{1}\right) u^{\prime}=\left(a_{0}, a_{1}\right)\left(b_{0}, b_{1}\right) u^{\prime \prime},
$$

$u^{\prime}, u^{\prime \prime} \in(A, B)$. The least trivial computation needed to obtain this result is

$$
\left(b_{1},\left(a_{1}, a_{0} b_{0}\right)\right) \in\left(B,{ }_{1} A\right) \leqq{ }_{1}(A, B) \leqq(A, B) .
$$

Applying Lemma 1 over again (i.e., letting $u_{0}=q$ and $u_{1}=t$ ),

$$
\left(a_{0} b_{0} u_{0}, a_{1} b_{1} u_{1}\right)=\left(a_{0}, a_{1}\right)\left(b_{0}, b_{1}\right) u, \quad u \in(A, B) .
$$

Again use has been made of the fact that $(A, B)$ is normal.

Suppose true for $k$; we want to show that

$$
\left({ }^{k} a^{k} b v, a b u\right)={ }^{k+1} a^{k+1} b w
$$

where $v \in \in_{k-1}(A, B), u \in(A, B), w \in \in_{k}(A, B),{ }^{m} a \in{ }^{m} A,{ }^{m} b \in{ }^{m} B(m=k, k+1)$. As before, we apply Lemma 1 first to

$$
\left({ }^{k} a^{k} b, a b\right)
$$


to obtain $\left({ }^{k} a^{k} b, a b\right)={ }^{k+1} a^{k+1} b w^{\prime}, w^{\prime} \in \in_{k}(A, B)$. Here again we have used Theorems 3.4 and 3.7. Now again apply Lemma 1 to

$$
\left({ }^{k} a^{k} b v, a b u\right), \quad v \in{ }_{k-1}(A, B), \quad u \in(A, B),
$$

with $v=q$ and $u=t$. This gives

$$
\left({ }^{k} a^{k} b v, a b u\right)={ }^{k+1} a^{k+1} b w, \quad w \in{ }_{k}(A, B) .
$$

Here Theorem 3.1 is useful for carrying out computations, e.g.

$$
\begin{aligned}
& \left({ }^{k} a^{k} b, u\right) \in\left({ }_{k} G,(A, B)\right) \leqq{ }_{k+1}(A, B) \leqq{ }_{k}(A, B), \\
& \left(u,\left(a b,{ }^{k} a^{k} b v\right)\right) \leqq\left((A, B),{ }_{k+1} G\right) \leqq{ }_{k}(A, B) .
\end{aligned}
$$

This is sufficient to prove Lemma 2.

Proof of Theorem. $\left({ }^{m} G,{ }^{n} G\right) \cap(A, B) \geqq\left({ }_{m} A,{ }_{n} B\right)\left({ }_{n} A,{ }_{m} B\right)$ obviously. We want to prove the opposite inequality. By definition, if $x \in\left({ }^{m} G,{ }^{n} G\right)$, then

$$
x=\prod_{i=1}^{r}\left({ }^{m} g_{i},{ }^{n} g_{i}\right), \quad \text { where } \quad{ }^{m} g_{i} \in{ }^{m} G, \quad{ }^{n} g_{i} \in{ }^{n} G .
$$

By Lemma 2, ${ }^{m} g_{i}={ }^{m} a_{i}{ }^{m} b_{i}{ }^{m-1} u_{i},{ }^{n} g_{i}={ }^{n} a_{i}{ }^{n} b_{i}{ }^{n-1} u_{i}$, for some ${ }^{k} a_{i} \in{ }^{k} A,{ }^{k} b_{i} \in{ }^{k} B$, ${ }^{k} u_{i} \in \in_{k}(A, B), k=m, n, m-1, n-1$. Using Lemma 1 twice, and the same procedure as in the proof of Lemma 2, we obtain

$$
\left({ }^{m} g_{i},{ }^{n} g_{i}\right)=\left({ }^{m} a_{i},{ }^{n} a_{i}\right)\left({ }^{m} b_{i},{ }^{n} b_{i}\right) u, \quad u \in\left({ }_{n} A,{ }_{m} B\right)\left({ }_{m} A,{ }_{n} B\right) .
$$

Here use is made of the fact that $\left({ }_{n} A,{ }_{m} B\right)\left({ }_{m} A,{ }_{n} B\right)$ is normal and of Theorem 3.1 ; e.g.

$$
\left({ }^{n} b_{i},\left({ }^{n} a_{i},{ }^{m} a_{i}{ }^{m} b_{i}\right)\right) \leqq\left({ }_{n} B,\left({ }_{n} A,{ }_{m} G\right)\right) \leqq\left({ }_{n} B,{ }_{m} A\right) .
$$

Repeated use is also made of $\mathrm{G} I ; 2.1 .2$ and $\mathrm{G} \mathrm{I} ; 2.1 .3$.

Thus if $x \in\left({ }^{m} G,{ }^{n} G\right)$, then

$$
x=\prod_{i=1}^{r}\left({ }^{m} a_{i},{ }^{n} a_{i}\right)\left({ }^{m} b_{i},{ }^{n} b_{i}\right) v, \quad v \in\left({ }_{n} A,{ }_{m} B\right)\left({ }_{m} A,{ }_{n} B\right) .
$$

If $x \in(A, B)$, then

$$
\prod_{i}\left({ }^{m} a_{i},{ }^{n} a_{i}\right)=\prod_{i}\left({ }^{m} b_{i},{ }^{n} b_{i}\right)=1
$$

by the unique representation theorem, G II ; 1.2 . Since $\left(\left({ }^{m} a_{i},{ }^{n} a_{i}\right),\left({ }^{m} b_{i},{ }^{n} b_{i}\right)\right)$ $\in\left({ }_{n} A,{ }_{m} B\right)$, we can "shuffle" the $\left({ }^{m} a_{i},{ }^{n} a_{i}\right)$ and $\left({ }^{m} b_{i},{ }^{n} b_{i}\right)$ around (by means of the identity $p q=q p(p, q))$, so that if $x \in\left({ }^{n} G,{ }^{m} G\right) \cap(A, B)$, we obtain $x \in\left({ }_{n} A,{ }_{m} B\right)\left({ }_{m} A,{ }_{n} B\right)$. q.e.d.

Comment. For further details, see Moran's paper.

THEOREM 6.3. Let $k$ be a fixed non-negative integer, $G=A * B$, 
$A \circ B=\frac{A * B}{\mathfrak{R}[D(k)]}, \quad D(k)=\left\{\begin{array}{l}(a, b), a \in A, b \in B, \text { such that either } \\ a \text { is in the center of } A \text { and } a^{k}=1 \text { or } \\ b \text { is in the center of } B \text { and } b^{k}=1\end{array}\right\}$.

Then $\circ$ is a fully regular product which does not satisfy MacLane's postulate; hence it is not a verbal product.

The following lemma will be needed in the proof of Theorem 6.3:

Lemma 3. $(A, B)$ in $A \circ B$ is a free group; in particular, $(A, B)$ is generated by $(a, b), a \in A, b \in B$, but some of the $(a, b)$ are identified, i.e.,

$$
\left(a_{i} a, b_{i} b\right)=\left(a a_{i}, b_{i} b\right)=\left(a_{i} a, b b_{i}\right)=\left(a a_{i}, b b_{i}\right)=\left(a_{i}, b_{i}\right)
$$

for all $a \in A_{k}=\left\{a, a \in\right.$ center of $A$ and $\left.a^{k}=1\right\}$ and all $b \in B_{k}=\{b, b \in$ center of $B$ and $\left.b^{k}=1\right\}$. A part from these identities, there are no other relations which hold among the generators of $(A, B)$; in particular, there are no elements of finite order.

Proof of lemma. It is sufficient to show that the only elements of $(A, B)$ in $A \circ B$ which equal 1 are the following: Let $u=\prod_{i=1}^{n}\left(a_{i}, b_{i}\right)^{k_{i}}$ be an element of $(A, B)$ which equals 1 . If $x_{i}=\left(a_{i}, b_{i}\right)$ and the identities indicated in (1) are made; e.g., if $x_{5}=\left(a_{2} a, b_{2}\right), a \in A_{k}$, then let $x_{5}$ be replaced by $x_{2}$ in the expression for $u$ in terms of the $x_{i}$; then the expression for $u$ becomes an identity in the free group generated by the $x_{i}$. It is sufficient to show that this is true for all the elements of $A * B$ which equal 1 in $A \circ B$.

Any element in $(A, B)$ of $A * B$ which equals 1 in $A \circ B$ is a product of elements of the form

$$
g^{-1}(a, b) g
$$

where $g \in A * B$ and either $a \in A_{k}$ or $b \in B_{k}$. Let $g=a_{1} b_{1} a_{2} b_{2} \cdots a_{n} b_{n}$. The argument will be by induction on the length of $g$. If $g$ is of length 1 , then by use of the identities

$$
u^{-1}(v, w) u=(v u, w)(w, u)=(u, v)(v, w u) \quad(\text { for any } u, v, w)
$$

we obtain

$$
\begin{aligned}
& a_{1}^{-1}(a, b) a_{1}=\left(a a_{1}, b\right)\left(b, a_{1}\right), \\
& b_{1}^{-1}(a, b) b_{1}=\left(b_{1}, a\right)\left(a, b b_{1}\right) .
\end{aligned}
$$

Hence, if we let $x_{1}=\left(a_{1}, b\right), y_{1}=\left(b_{1}, a\right)$, and we use (1), then (3) and (4) become

$$
x_{1} x_{1}^{-1}, \quad y_{1} y_{1}^{-1} \text { or } 1 \cdot 1
$$

depending on whether $a \in A_{k}$ or $b \in B_{k}$. Suppose the lemma is true for $g$ of length $n-1$, i.e., $g^{-1}(a, b) g=\prod_{i=1}^{m}\left(a_{i}, b_{i}\right)^{k_{i}}$ where, if $x_{i}$ are substituted for the 
$\left(a_{i}, b_{i}\right)$ as indicated by (1), then $\prod_{i} x_{i}=1$ as a product in the free group generated by the $x_{i}$. For every $\left(a_{i}, b_{i}\right)$ such that $a_{i} \notin A_{k}$ and $b_{i} \notin B_{k}$, there corresponds either $\left(b_{i}, a a_{i}\right)$ or $\left(b b_{i}, a_{i}\right)$ with $a \in A_{k}, b \in B_{k}$. Then using (2),

$$
\begin{aligned}
a_{n}^{-1}\left(a_{i}, b_{i}\right) a_{n} & =\left(a_{i} a_{n}, b_{i}\right)\left(b_{i}, a_{n}\right)=x_{n} y_{n}, \\
a_{n}^{-1}\left(b_{i}, a a_{i}\right) a_{n} & =\left(a_{n}, b_{i}\right)\left(b_{i}, a a_{i} a_{n}\right)=y_{n}^{-1} x_{n}^{-1}, \\
a_{n}^{-1}\left(b b_{i}, a_{i}\right) a_{n} & =\left(a_{n}, b b_{i}\right)\left(b b_{i}, a_{i} a_{n}\right)=y_{n}^{-1} x_{n}^{-1} .
\end{aligned}
$$

Similar identities hold with $b_{n}$ substituted for $a_{n}$. Therefore, if $g^{-1}(a, b) g$ $=\prod_{i}\left(a_{i}, b_{i}\right)^{k_{i}}$ has the properties mentioned above, so does

$$
a_{n}^{-1} g^{-1}(a, b) g a_{n} \text { and } b_{n}^{-1} g^{-1}(a, b) g b_{n} . \quad \text { q.e.d. }
$$

Proof of theorem. That this product does not satisfy MacLane's postulate can be seen readily by letting $A$ and $B$ be the additive group of integers and $M$ the integral multiples of $k$ and $N$ the indentity. Then

$$
\frac{A \circ B}{M N}
$$

is the free product of the integers modulo $k$ and the integers, while

$$
\frac{A}{M} \circ \frac{B}{N}
$$

is the direct product of the integers modulo $k$ and the integers. Since verbal products satisfy MacLane's postulate, this product cannot be verbal.

That $\mathrm{o}$ is commutative is trivial. Associativity is the only difficult matter:

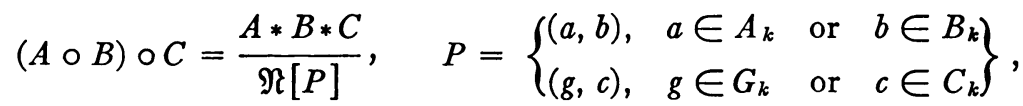

$$
\begin{aligned}
& A \circ(B \circ C)=\frac{A * B * C}{\mathfrak{N}[Q]}, \quad Q=\left\{\begin{array}{llll}
(b, c), & b \in B_{k} & \text { or } & c \in C_{k} \\
(a, e), & a \in A_{k} & \text { or } & e \in E_{k}
\end{array}\right\},
\end{aligned}
$$

where $G=A * B, E=B * C$, and

$$
\begin{array}{rr}
R_{k} & =\left\{r \in \text { center of } R \text { and } r^{k}=1\right\}, \quad \text { for } R=A, B, C ; \\
& =\left\{r \in \text { center of } A \circ B \text { and } r^{k}=1 \text { considering } r \text { as an element of } A \circ B\right\}, \\
& =\left\{r \in \text { center of } B \circ C \text { and } r^{k}=1 \text { considering } r \text { as an element of } B \circ C\right\}, \\
\text { for } R=A * B ;
\end{array}
$$

It is sufficient to show that $\mathfrak{R}[Q] \leqq \mathfrak{N}[P]$. That $(b, c) \in P$ for $b \in B_{k}$ or $c \in C_{k}$ follows from the fact that $b \in B_{k}$ implies $b \in G_{k}$. Now consider $(a, e), a \in A_{k}$. 
By G I ; 2.1.3 and induction on the length of $e$ as an element in $B * C,(a, e)$ $\in \mathfrak{N}[P]$.

For $(a, e), e \in E_{k}$, the argument is somewhat more complicated. Let $\boldsymbol{e}=b c u, b \in B, c \in C, u \in(B, C)$. Since $e^{k}=b^{k} c^{k} v, v \in(B, C)$ by unique representation (G II ; 1.2), and since $e \in E_{k}$, we obtain $b^{k}=c^{k}=1$. Since $e$ as an element of $B \circ C \in$ center of $B \circ C$,

$$
b_{1} c_{1} u_{1} b c u=b c u b_{1} c_{1} u_{1}
$$

for all $b_{1} \in B, c_{1} \in C, u_{1} \in(B, C)$ of $B \circ C$. By unique representation, $b \in$ center of $B, c \in$ center of $C$. Thus $b$ and $c \in$ center of $B \circ C$, and hence $u \in$ center of $B \circ C$. Hence in $B \circ C, e^{k}=u^{k}=1$. But by Lemma $3,(B, C)$ is free in $B \circ C$, and hence $u=1$ in $B \circ C$, and hence $u=1$ in $(A \circ B) \circ C$. Therefore, $(a, e)$ $e \in E_{k}$ is of the form $(a, b c)$ in $(A \circ B) \circ C, b \in B_{k}, c \in C_{k}$. G I; 2.1.3 shows that $(a, b c)=1$ in $(A \circ B) \circ C$. Hence $(a, e)=1$ in $(A \circ B) \circ C$. Hence $(a, e) \in \mathfrak{R}[P]$. q.e.d.

SUMMARY OF PRODUCTS

$\mathrm{A} \circ B=A * B / R$

\begin{tabular}{|c|c|c|}
\hline$R$ & Theorem & Associative? \\
\hline$(\mathfrak{R}[(A, A)], \mathfrak{N}[B])$ & 4.1 & No \\
\hline $\mathfrak{N}[((A, A),(B, B))]$ & 4.2 & No \\
\hline$((A, B),(A, B))$ & 4.3 & No \\
\hline $\mathfrak{R}\left[\left\{x^{2}, x \in(A, B)\right\}\right]$ & 4.4 & No \\
\hline $\mathfrak{R}\left[\left\{(a, b)^{2}, a \in A, b \in B\right\}\right]$ & 4.5 & No \\
\hline $\mathfrak{N}[\{(a b a b, g), a \in A, b \in B, g \in A * B\}] \cap(A, B)$ & 4.6 & No \\
\hline$((A, A * B),(B, A * B)) \cdot \mathfrak{R}[\{(a b a b, g), a \in A, b \in B, g \in A * B\}] \cap(A, B)$ & 4.7 & No \\
\hline$((A, B),(A * B, A * B))$ & 4.8 & No \\
\hline$((\mathfrak{N}[A], \mathfrak{N}[A]),(\mathfrak{N}[B], \mathfrak{N}[B]))$ & 4.9 & No \\
\hline$k((A, B),(A, B)), k \geqq 0$ & 4.10 & No \\
\hline ((A, $A * B),(B, A * B)), k \geqq 1$ & 4.11 & No \\
\hline$\left({ }_{m} A,{ }_{n} B\right)\left({ }_{n} A,{ }_{m} B\right)$ & 5.1 & Yes \\
\hline$\prod_{i=1}^{k}\left({ }_{m_{i}} A,{ }_{n_{i}} B\right)\left({ }_{n_{i}} A,{ }_{m_{i}} B\right)$ & 5.5 & Yes \\
\hline$\left\{\begin{array}{cl}(a, b), & a \in \text { center of } A \text { and } a^{k}=1 \text { or } \\
& b \in \text { center of } B \text { and } b^{k}=1 . \\
& k \text { is a fixed integer }\end{array}\right\}$ & 6.3 & Yes \\
\hline
\end{tabular}


CHART OF PRODUCTS

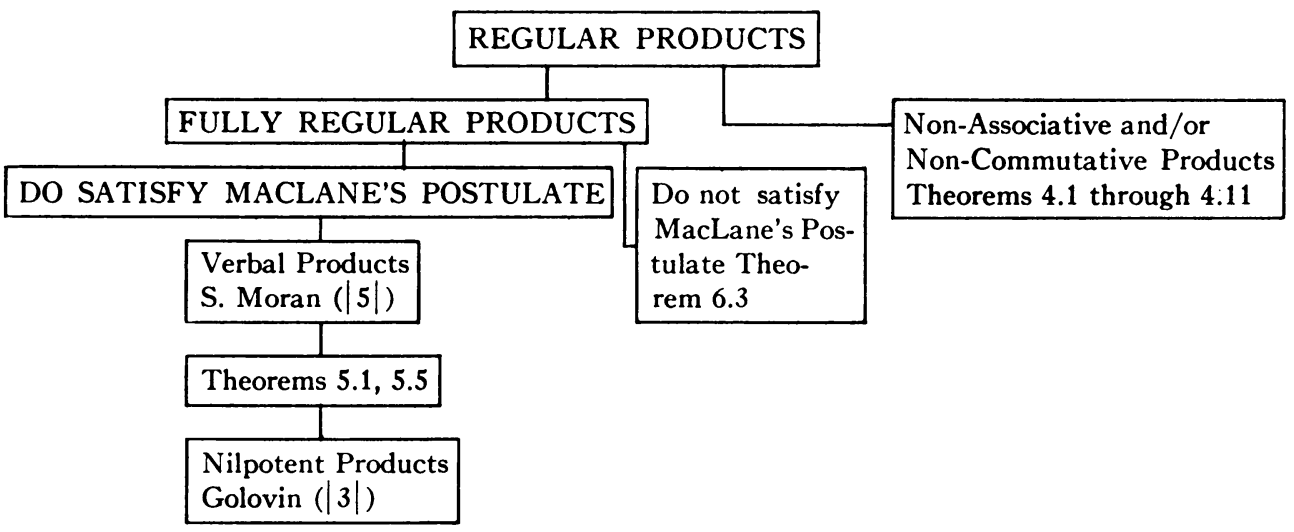

\section{BIBLIOGRAPHY} 1953.

1. A. G. Kurosh, Gruppentheorie (trans. from the Russian), Berlin, Akademie-Verlag,

2. B. L. van der Waerden, Modern algebra (trans. from the German), New York, Ungar, 1949.

3. O. N. Golovin, Nilpotentnii Proiszvedeniya Grup (Nilpotent products of groups), Mat. Sbornik N.S. vol. 27 (69) No. 3 (1950) pp. 427-454. American Mathematical Society Translations, ser. 2, vol. 2, 1956, pp. 89-115.

4. W. Magnus, Über Beziehungen zwischen höheren Kommutatoren, J. Reine Angew. Math. vol. 177 (1937) pp. 105-115.

5. S. Moran, Associative operations on. groups, Doctoral thesis, London, 1954.

New York University,

NEW YORK, N. Y. 